\title{
Cluster observations of reconnection due to the Kelvin-Helmholtz instability at the dawnside magnetospheric flank
}

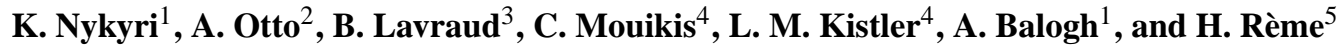 \\ ${ }^{1}$ The Blackett Laboratory, Imperial College, London, UK \\ ${ }^{2}$ Geophysical Institute, University of Alaska, Fairbanks, USA \\ ${ }^{3}$ Los Alamos National Laboratory, New Mexico, USA \\ ${ }^{4}$ University of New Hampshire, USA \\ ${ }^{5}$ Centre d'Etude Spatiale Rayonnements, Toulouse, France
}

Received: 24 October 2005 - Revised: 22 August 2006 - Accepted: 24 August 2006 - Published: 20 October 2006

\begin{abstract}
On 3 July 2001, the four Cluster satellites traversed along the dawnside magnetospheric flank and observed large variations in all plasma parameters. The estimated magnetopause boundary normals were oscillating in the $z$-direction and the normal component of the magnetic field showed systematic $\sim 2-3$ min bipolar variations for $1 \mathrm{~h}$ when the IMF had a small positive $b_{z}$-component and a Parker-spiral orientation in the $x, y$-plane. Brief $\sim 33 \mathrm{~s}$ intervals with excellent deHoffman Teller frames were observed satisfying the Walén relation. Detailed comparisons with 2D MHD simulations indicate that Cluster encountered rotational discontinuities generated by Kelvin-Helmholtz instability. We estimate a wave length of $\sim 6 R_{E}$ and a wave vector with a significant $z$-component.
\end{abstract}

Keywords. Magnetospheric physics (Magnetopause, cusp and boundary layers; Plasma waves and instabilities) - Space plasma physics (Magnetic reconnection; transport processes; Turbulence)

\section{Introduction}

The entry of solar wind plasma into the magnetosphere during quiet geomagnetic conditions and primarily northward IMF has been a puzzle for some time. Solar wind plasma penetration and transport has been proposed to arise from double high-latitude reconnection (Song and Russell, 1992; Russell et al., 2000; Onsager et al., 2001; Li et al., 2005; Oieroset et al., 2005; Lavraud et al., 2005) and diffusive processes such as ion mixing due to Kelvin-Helmholtz instability (KHI) (Fujimoto and Terasawa, 1994, 1995; Thomas and Winske, 1993; Hasegawa et al., 2004). These models have not been investigated in a quantitative fashion as to whether

Correspondence to: K. Nykyri

(k.nykyri@ic.ac.uk) they can lead to the observed efficient filling of Earth's magnetotail with cold, dense solar wind material.

Northward IMF conditions have long been associated with the presence of the cold, dense plasma sheet (CDPS) (Fairfield et al., 1981; Lennartsson, 1992). Borovsky et al. (1998) demonstrated that the plasma sheet temperature and density are correlated to the solar wind properties on a time scale of 1 to $2 \mathrm{~h}$. Terasawa et al. (1997) and Fujimoto et al. $(1998,2000)$ reported a strong correlation between the CDPS and northward IMF orientation during the hours prior to the plasma sheet observations.

MHD simulations by Otto and Fairfield (2000) - motivated by Geotail observations of the KHI (Fairfield et al., 2000) indicated that reconnection can occur inside the narrow current layers generated by the KHI at the flank magnetopause. Nykyri and Otto $(2001,2004)$ quantified this reconnection process inside $\mathrm{KH}$ vortices in two dimensions using MHD and Hall-MHD approximations. Their results indicate that reconnection inside $\mathrm{KH}$ vortices can transport plasma of solar wind origin into the magnetosphere with a transport velocity of $\sim 1.5 \mathrm{~km} / \mathrm{s}$. This corresponds to a diffusion coefficient of order $10^{9} \mathrm{~m}^{2} / \mathrm{s}$ which is sufficient to produce the LLBL at the flanks of the magnetopause during northward IMF conditions on the observed timescales (Fujimoto et al., 1998). Brackbill and Knoll (2001) used 3-D MHD simulations in idealized perpendicular configuration and found that KHI could cause reconnection on the KH time scale.

There are several ionospheric and ground observations (Lee and Olson, 1980; Olson and Rostoker, 1978; Olson, 1986; Ohtani et al., 1999) associated with the KHI as well as in-situ satellite observations (Fairfield et al., 2000; Nikutowski et al., 2002; Nykyri et al., 2003; Hasegawa et al., 2004), both from the dawn and dusk-side flanks boundary layer. The most recent of these (Hasegawa et al., 2004) used multi-point Cluster satellite observations at the duskside lowlatitude flank magnetopause at $x \sim-3 R_{E}$. Hasegawa et al. (2004) could demonstrate the non-linear rolled-up nature of

Published by Copernicus GmbH on behalf of the European Geosciences Union. 
the vortices. However they didn't observe signatures of reconnection.

In the present paper we use 4-point Cluster measurements close to the equatorial $\left(z \sim 4 R_{E}\right)$, near tail $\left(x<-8 R_{E}, y<-\right.$ $16 R_{E}$ ), dawnside flank magnetopause on 3 July 2001. During this day Cluster spacecraft had several quasi-periodic encounters with magnetospheric and magnetosheath-type plasmas. We will mostly focus here on the interesting subinterval between 05:00-06:00 UT during which the IMF was mostly in Parker-Spiral configuration with a small positive or nearly zero $b_{z}$-component. We will also discuss the overall features and differences observed in comparison to the subsequent interval 07:45-08:45 UT during which the IMF conditions have changed but the large-scale plasma variations occasionally show similar features as during the earlier interval. Between 05:00-06:00 UT Cluster encounters a strongly perturbed magnetopause with a continuous chain of bipolar magnetic field variations. Cluster also recorded brief intervals of plasma mixing for which deHoffmann-Teller (HT) frames are well defined and the Walén relation is satisfied. We interpret these to be signatures of reconnection embedded in $\mathrm{KH}$-vortices that have $k$-vectors out from the equatorial plane. Cluster observations on this day may indicate that plasma transport from the magnetosheath into the magnetosphere due to reconnection driven by KHI may be possible not only for strongly northward IMF but also for other IMF orientations.

\section{Instrumentation and data analysis tools}

We use data from two instruments onboard Cluster. High resolution (22.4 vectors/second) and spin averaged (4s) magnetic field measurements are obtained from the Flux Gate Magnetometer (FGM) (Balogh et al., 1997, 2001) from all four spacecraft. Ion plasma measurements were obtained using the Cluster Ion Spectrometry (CIS) instruments (Rème et al., 2001). We make use of the temperature, velocity and density from the Hot Ion Analyser (HIA) on board spacecraft 1 and 3, at spin resolution $(\sim 4 \mathrm{~s})$. The proton velocity and densities for sc 4 are obtained from the ion COmposition and DIstribution Function analyser (CODIF) for every $\sim 8 \mathrm{~s}$.

Our data analysis tools use the deHoffman-Teller (HT) analysis and the Walén relation (Sonnerup et al., 1995). The boundary normals are calculated using Minimum Faraday Residue (MFR) method (Khrabrov and Sonnerup, 1998). Haaland et al. (2004) used this method and compared it with other single- and multi-spacecraft methods for the magnetopause event on 5 July 2001. They found that multispacecraft technique Constant Thickness Approach (CTA) and a hybrid technique CTAM gave reasonably good agreement $\left(\sim 5^{\circ}\right)$ with MFR-method and with the MVABC (Minimum Variance Analysis of the Magentic field-method using additional constraint that the normal component of the magnetic field, $B_{N}$, is zero)-method. The MVAB method with- out the constraint did not perform well during their event. Here we could not use the MVABC method because the magnetic field had significant normal components between 05:00-06:00 UT. We chose the MFR-method over the multispacecraft techniques in order to automate the normal calculations using a sliding window method. For many intervals the transitions of the boundaries occur nearly simultaneously at all 4 spacecraft so that the multi-spacecraft techniques would have failed.

The HT frame is a frame where the convection electric field vanishes, thus indicating an approximately steady state plasma configuration. The HT velocity, $\boldsymbol{v}_{H T}$, is determined by minimising $\left|\left(\boldsymbol{v}-\boldsymbol{v}_{\text {obs }}\right) \times \boldsymbol{B}_{\text {obs }}\right|^{2}$ in terms of the constant transformation velocity $v$ for a given dataset (Sonnerup et al., 1995).

The Walen relation is calculated in the HT frame as $\boldsymbol{v}-v_{H T}= \pm C \boldsymbol{v}_{A}$ and implies that in the HT-frame the plasma flow velocity is Alfvénic (Sonnerup et al., 1995). The Alfvén velocity, $\boldsymbol{v}_{A}=\boldsymbol{B} / \sqrt{\left(\mu_{0} n_{p} m_{p}\right)}$, is corrected by factor $C=\sqrt{(1-\alpha)}$, where $\alpha=\left(T_{\|}-T_{\perp}\right) n_{p} k_{B} \mu_{0} / B^{2}$ is the pressure anisotropy correction (e.g. Sonnerup et al., 1981). The Walén relation is satisfied for Alfvén waves, rotational discontinuities, but also approximately for intermediate and switch-off slow shocks. The latter two are often associated with magnetic reconnection.

\section{Solar wind conditions and overview of the event}

Figure 1 shows an overview plot of Cluster plasma observations by sc1 (top 3 panels) and solar wind conditions measured by ACE (bottom 2 panels) at L1. The top panel shows the omni-directional ion spectrogram (energy flux). The second panel shows the ion number density and the third panel shows the ion temperature. The second lowest panel shows solar wind RAM pressure (red) and solar wind velocity (blue), and the bottom panel represents IMF components. The ACE data has been lagged $68 \mathrm{~min}$ to take into account the finite propagation speed of the solar wind from L1 to Cluster location.

The solar wind properties can be roughly divided into two types of intervals: Between 03:00-08:00 UT the interplanetary magnetic field has a Parker-spiral orientation in $x, y$-plane with small, mostly positive $b_{z}$-component until 07:40 UT. After 07:40 UT IMF $b_{z}$ turns negative, and after 08:00 UT also $b_{x}$ turns negative, forming an orthoParker spiral. Solar wind dynamic pressure is varying between $1.5-3.7 \mathrm{nPa}$ and speed between $380-440 \mathrm{~km} / \mathrm{s}$. Cluster spacecraft are traversing the tail flank close to the equatorial dawnside $\left(r \sim[-9,-17,4] R_{E}\right)$ and have several encounters with high density and low temperature magnetosheathlike, and tenuous and hot magnetospheric-like plasma. Prior to 04:30 UT Cluster is in the tenuous magnetospheric-type plasma with ion energies typically between $1000-10000 \mathrm{eV}$. As solar wind pressure gradually doubles from $1.5 \mathrm{nPa}$ to 


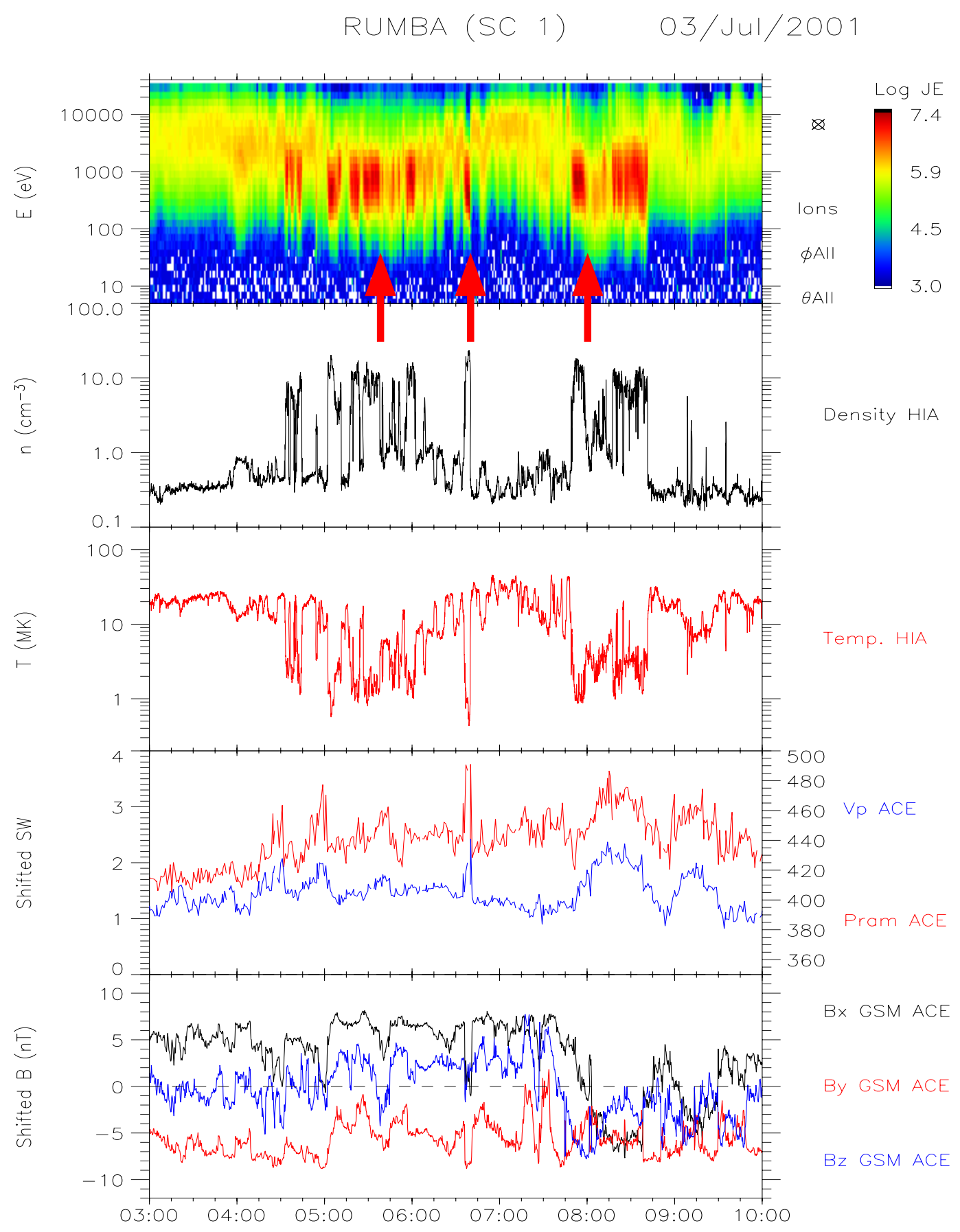

Fig. 1. Overview plot of Cluster spacecraft 1 (sc1) and ACE (lagged) magnetic field and plasma data on 3rd of July 2001 between 03:0010:00 UT. The panels from top to bottom show i) omni-directional energy flux spectrogram for ions, ii) ion density, iii) ion temperature iv) proton velocity and dynamic pressure at ACE, vii) and magnetic field at ACE. Red arrows mark the start of the intervals when magnetosheath energy ions are observed in tenuous plasma.

$3 \mathrm{nPa}$ the magnetopause is pushed inwards and Cluster encounters higher density magnetosheath plasma, with ion energies of order $\sim 200-3000 \mathrm{eV}$. The pressure then slightly decreases and Cluster moves back into the magnetosphere. Similar encounters are observed again at $\sim 05: 00$ UT when there is another pressure enhancement. Between 05:0006:37 UT the pressure is fairly constant showing a gradual increase of order 20 percent followed by a drop at $\approx 05: 45$ UT but throughout this interval there are several quasi-periodic encounters of magnetosheath (MSH) and mixed boundary 

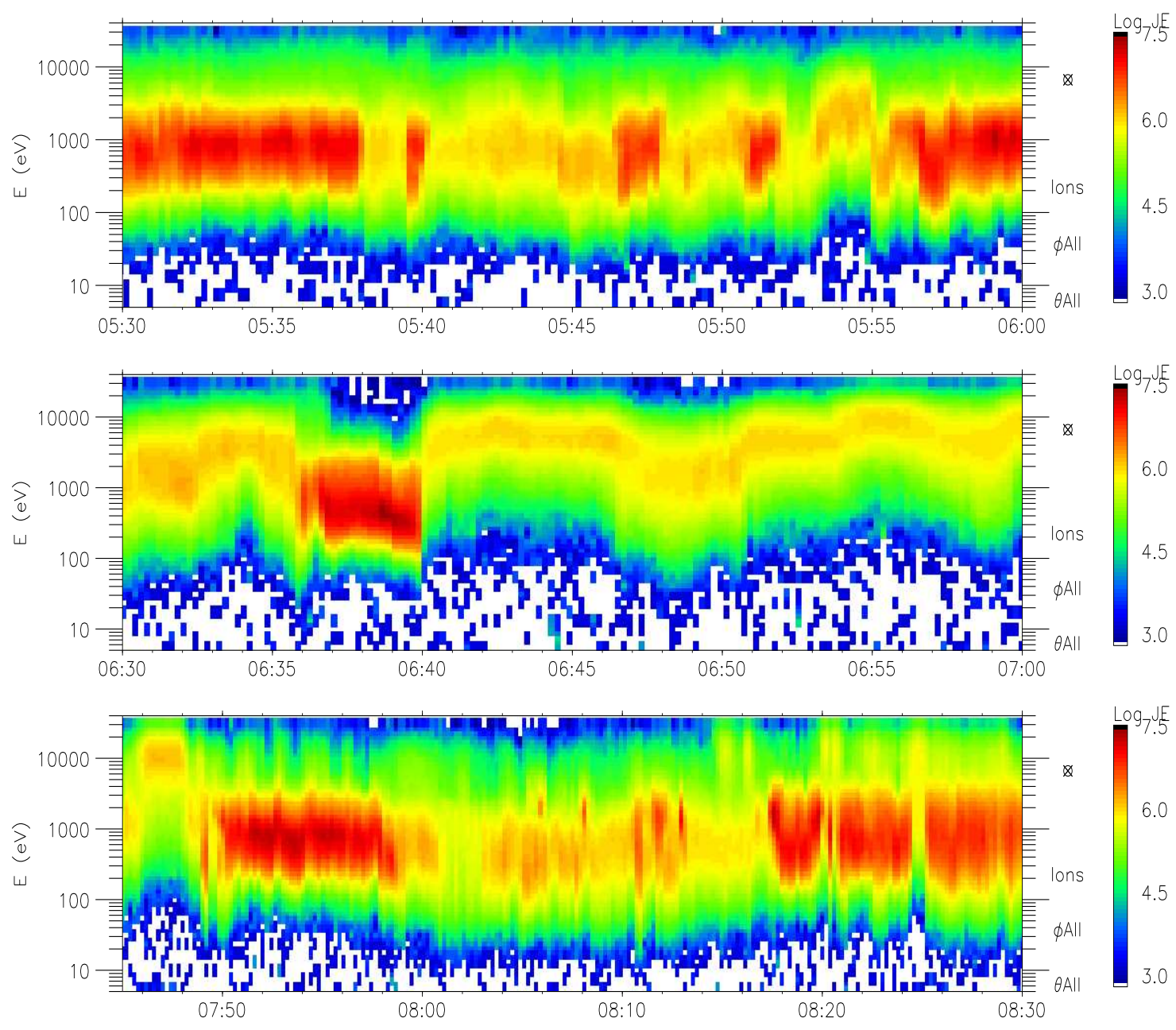

Fig. 2. Omni-directional energy flux spectrogram for ions at sc1 at three intervals (start of the interval corresponding to the red arrows at Fig. 1): 05:30-06:00 UT (top panel), 06:30-07:00 UT (middle), 07:45-08:30 UT (bottom panel).

layer type of plasma (ion energies are magnetosheath-like but density much lower than magnetosheath values). For example the sub-interval between 05:37-05:56 UT (first red arrow) has patches of relatively tenuous $\left(n=1 / \mathrm{cm}^{3}\right.$; for higher resolution see top left panel of Fig. 5) plasma with magnetosheath energy ions (top panel of Fig. 2). A pressure pulse moves the magnetopause inward over the spacecraft at $\approx 06: 37$ UT, which can be seen as an encounter of MSHtype plasma. At this time the energy fluxes of ions at $E>\approx 2000 \mathrm{eV}$ drop significantly (see second red arrow in Fig. 1 and close up in middle panel of Fig. 2). After this, the spacecraft are mostly in the magnetosphere up to $\approx 07: 40$ UT. At this time the IMF $b_{z}$ rotates southward and 15 min later is followed by the solar wind pressure enhancement and rotation of $b_{x}$-component so that the IMF in $x, y$-plane forms an ortho-Parker spiral. The increased pressure pushes the magnetopause closer to Cluster so that the spacecraft are swept into the magnetosheath at 07:47 UT. Interestingly, the patches of tenuous plasma with magnetosheath energies ob- served between 08:00-08:15 UT (see third red arrow in Fig. 1 and a close up in third panel of Fig. 2) resemble those of the earlier interval between 05:37-05:56 UT although the solar wind conditions have changed. Violation of the frozen in condition is needed to mix the plasma of these two regions. So the question is: what are the physical processes generating these mixed layers of plasma for these two different solar wind conditions? Between 08:15-08:45 UT Cluster has several encounters with magnetosheath plasma after which it moves into the magnetosphere for the remainder of the interval. On its next outbound leg on 4/5 July 2005 Cluster had another long encounter with the magnetopause at a similar location (Paschmann et al., 2005). Their determinations of the magnetopause orientation for 96 individual magnetopause crossings indicated evidence of surface waves.

Figure 3 shows Cluster tetrahedron formation in 4 different views at 05:00 UT on 3 July 2001: $x, y$-plane (top left), 30 degree rotation about $z$ - and $x$-axis (top right), $60 \mathrm{de}$ gree rotation about $z$-axis and 30 degree rotation about $x$-axis 


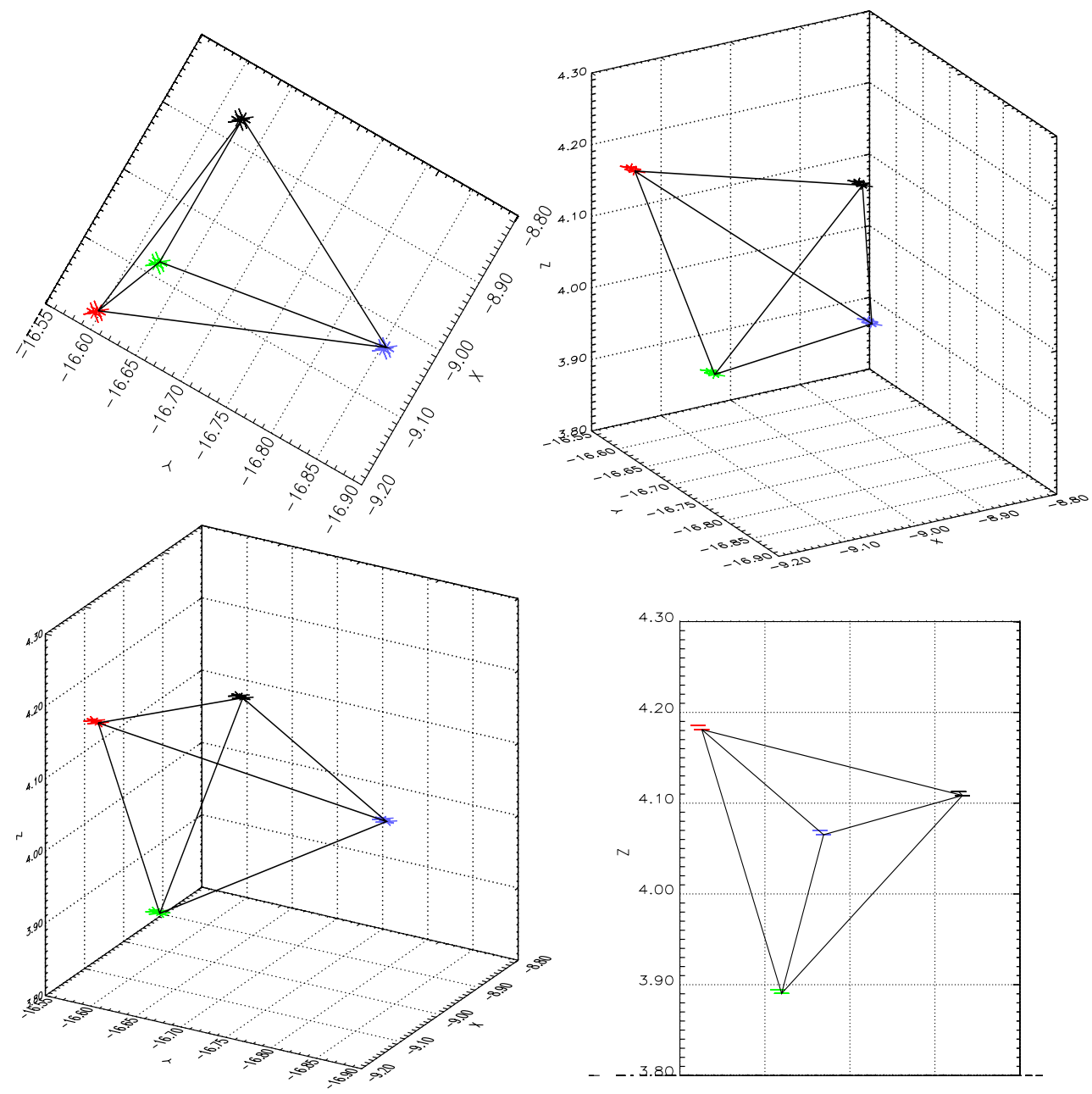

Fig. 3. Cluster constellation at 05:00 UT on 3 July 2001 from 4 different views in GSM coordinates: $x, y$-plane (top left), 30 degree rotation about $z$ - and $x$-axis (top right), 60 degree ration about $\mathrm{z}$-axis and 30 degree rotation about $x$-axis from (bottom left), view from $-y$-axis (bottom right). Black, red, green and blue colors represent sc1, sc2, sc3 and sc4, respectively.

from (bottom left), view from $-y_{\mathrm{GSM}}$-axis (bottom right). One can see that spacecraft four (sc4 hereafter) is located furthest to the $-y_{\mathrm{GSM}}$-direction, $\mathrm{sc} 1$ and $\mathrm{sc} 4$ are almost at the same $z$-plane and sc 3 is located closest to the equatorial plane.

Figure 4 shows Cluster plasma (left) and magnetic field (right) observations in GSM coordinates between 03:0010:00 UT (see caption for details). In addition, the spacecraft distances from sc3, projected along the boundary normal direction are given. Sc1 is separated $90 \mathrm{~km}, \mathrm{sc} 2$ is $-627 \mathrm{~km}$, and sc 4 is separated $1260 \mathrm{~km}$ along the normal direction from sc3. One can see that Cluster is located very close to the dawnside magnetospheric flank at $r=[-9,-16.7,4] R_{E}$.

The plasma data (from the left-side panel composer) shows from top to bottom plasma density, three velocity components, total velocity and plasma temperature. Densities and ion temperatures are shown for sc1 and sc3 only, while velocities are for sc1, sc3 and sc4. There is a longer time scale oscillation in plasma temperature and density but one can also clearly see that there are several quasiperiodic shorter time-scale encounters with magnetosheathtype higher density, lower temperature plasma and with more tenuous and hot magnetospheric-type plasma. In the $v_{x}$ component of plasma velocity one can see the same larger time-scale oscillation with most negative $v_{x}$-values of $-350 \mathrm{~km} / \mathrm{s}$ corresponding to larger density, cooler temperature regions which we interpret as encounters with magnetosheath. However, within these large scale oscillation there are also smaller-scale velocity variations. The velocity components $v_{y}$ and interestingly also $v_{z}$ show both bipolar variations.

On the right hand side the first four panels show the magnetic field components and magnitude measured by four spacecraft and the lowest panel shows the three components of the current density that are calculated from $\boldsymbol{J}=\frac{1}{\mu_{0}} \nabla \times \boldsymbol{B}$, where $\boldsymbol{B}$ is linearly interpolated between four 

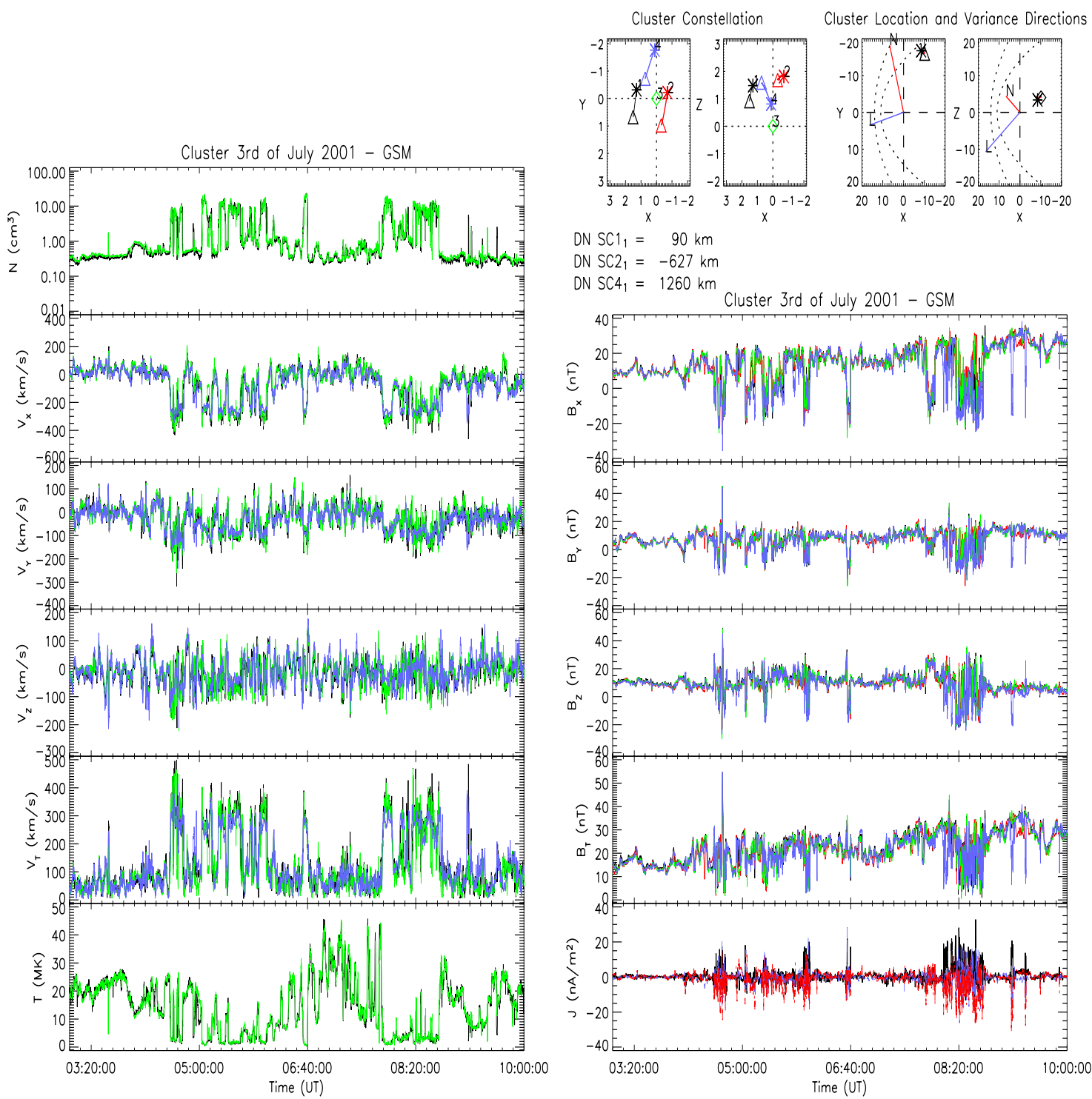

$\mathrm{DN} \mathrm{SC2} 2_{1}=-627 \mathrm{~km}$

$\mathrm{DN} \mathrm{SC} 4{ }_{1}=1260 \mathrm{~km}$

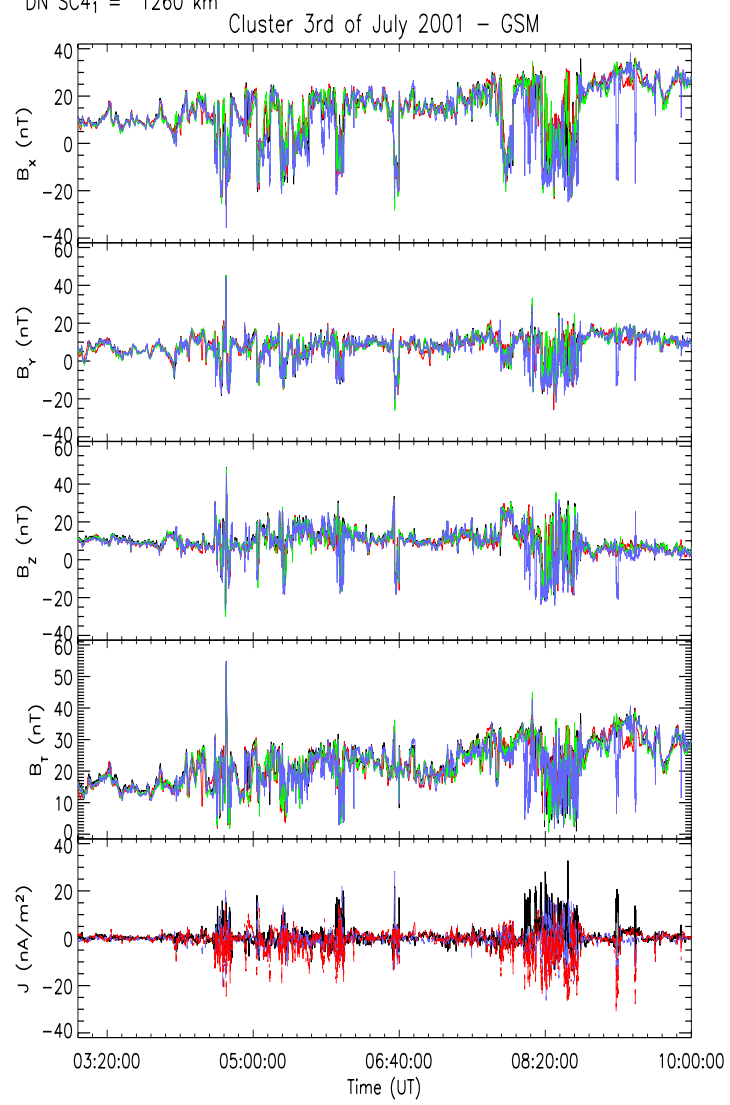

Fig. 4. Cluster plasma (left) and magnetic field observations (right) on 3 July 2001 between 03:20-10:00 UT. Black, red, green and blue colors represent sc1, sc2, sc3 and sc4 and their measurements, respectively. The first two panels in the upper right corner have Cluster constellation (asterisk marks the position at the beginning and diamond at the end of the interval) in $x, y$ - and $x, z$-planes in GSM coordinates relative to sc3 (in units of $1000 \mathrm{~km} / \mathrm{s}$ ). The last two panels in the upper right corner show Cluster location in $x, y$-and $x$, $z$-planes (GSM in units of $R_{E}$ ) with the approximate magnetopause and bow shock locations presented as parabolas. The boundary normal $(N)$ and tangent $(L)$ directions are shown as red and blue arrows (computed using sc1 data with MFR-method that is applied to the entire plotted interval), respectively. The plasma data shows from top to bottom ion density, three velocity components, total velocity and plasma temperature. On the right hand side the first four panels show the magnetic field components and magnitude and the lowest panel shows the three components of the current density (black, blue and red colors correspond to $x$-, $y$ - and $z$-components, respectively).

Cluster spacecraft. Thus, current layers with scales smaller than spacecraft separation are not resolved.

One can distinguish regions of negative $b_{x} \sim-15 \mathrm{nT}$ to $-20 \mathrm{nT}$ although in solar wind the $b_{x}$ is positive until $\sim 08$ :00 UT. These correspond to encounters of the magnetosheath field. When the IMF with positive $b_{x}$ and negative $b_{y}$ is draped around the magnetopause, a layer of negative tailward magnetic field component along the magnetospheric boundary (and thus a negative $b_{x}$ ) is formed on the dawnside. Another interesting feature is the strong $b_{z}$-component of up to $-20 \mathrm{nT}$ in the magnetosheath, which also could be generated due to the draping of IMF. The strength of this draped magnetic field along the shear flow layer determines whether this boundary becomes Kelvin-Helmholtz unstable or not. Nykyri et al. (2003) showed that magnetic reconnection and Kelvin-Helmholtz instability can show similar 

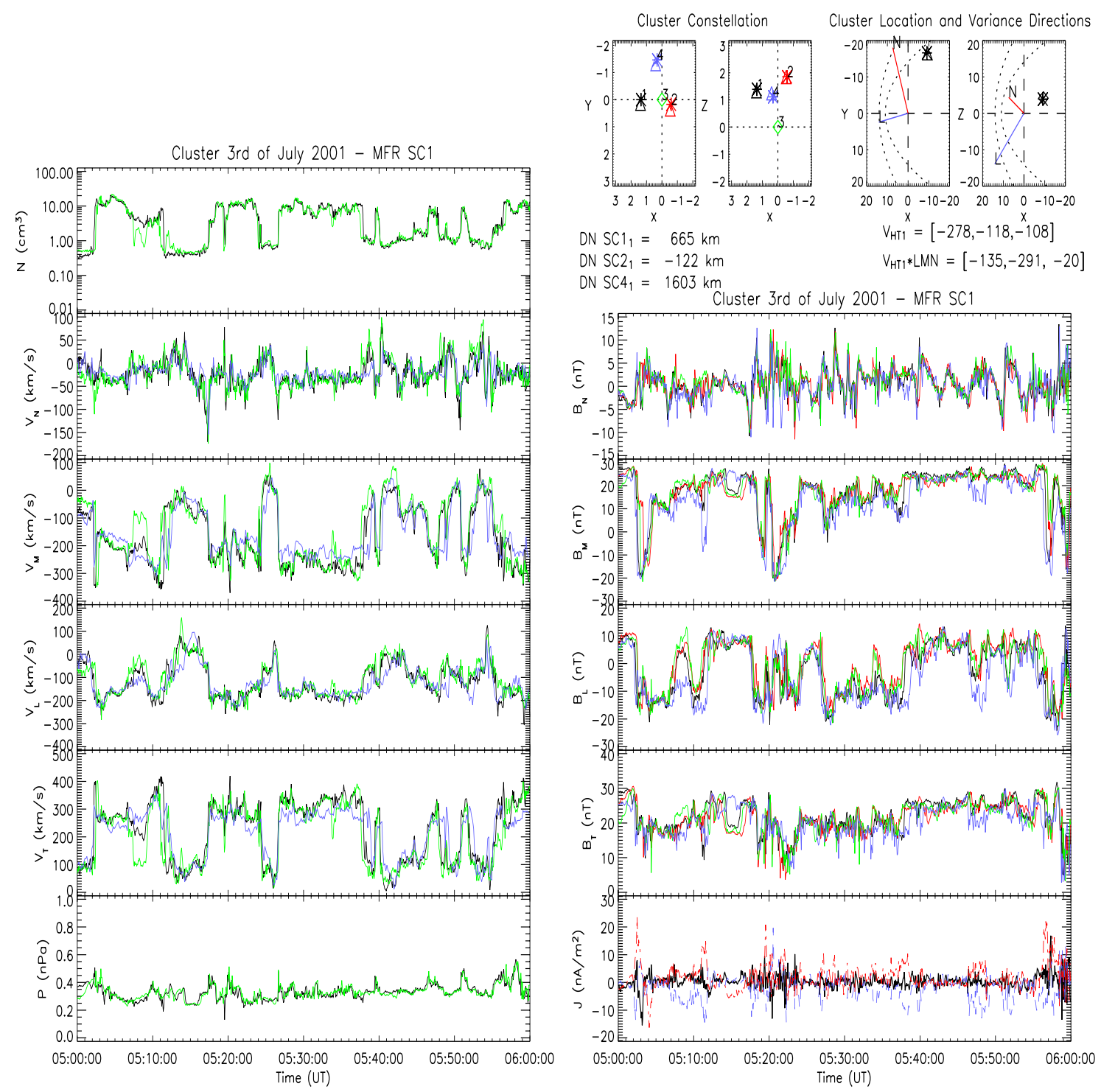

Fig. 5. Cluster plasma (left) and magnetic field observations (right) in $L M N$ coordinates determined by data of sc1 with MFR method on 3 July 2001 between 05:00-06:00 UT. Figure uses same format as Fig. 4, but instead of plasma temperature, the total pressure (ion plus magnetic) is shown in bottom left panel.

signatures in satellite data at the low-latitude boundary layer: both cause deformations of the boundary causing variations in the boundary normals and generating bipolar variations in the normal component of the magnetic field. If the KelvinHelmholtz has developed into its non-linear stage it can twist the magnetic field in the plane of the $\boldsymbol{k}$-vector of the instability resulting in anti-parallel magnetic field components and subsequently to reconnection (Nykyri and Otto, 2001, 2004) in the thin current sheets. If the satellite passes through such a region it should observe a good deHoffmann-Teller frame and Walén relation (Nykyri et al., 2003).

\section{Cluster observations between 05:00-06:00 UT during small positive $b_{z}$, Parker-Spiral IMF}

Figure 5 has the same layout as Fig. 4 (except instead of plasma temperature, the total pressure is shown) and shows Cluster plasma (left) and magnetic field (right) observations in local boundary normal coordinates $(L, M, N)$ of sc1 between 05:00-06:00 UT calculated with MFR method. The normal direction, $N$, is $[0.37,-0.90,0.22]$ and mostly in negative $y_{\mathrm{GSM}}$-direction. $L$ and $M$ are mutually orthogonal (and also orthogonal to $N$ ) and tangential to the boundary such that $L$ is $[0.71,0.12,-0.70]$ and is directed mostly sunward, mostly in positive $x_{\mathrm{GSM}}$-direction; $M$ is $[0.60,0.41,0.68]$ 
and completing the right-handed coordinate system pointing mostly to the positive $z_{\mathrm{GSM}}$-direction. The eigen values $\left[\lambda_{1}, \lambda_{3}, \lambda_{3}\right]$ are $[8.1,6.7,1.2]$, respectively, indicating that while the normal direction $N$ is well defined, the vectors $L$ and $M$ may be interchanged because $\lambda_{1} \approx \lambda_{2}$. During this interval the lagged ACE data shows mostly small positive $b_{z}$ of $\sim 4 \mathrm{nT}$ and Parker Spiral orientation. This IMF orientation leads to the anti-parallel magnetic field components in $x, y$-plane tangential to the shear flow boundary. The strong magnetosheath flow has a stabilising effect on the tearingmode in this plane, so that reconnection supposedly cannot operate in this plane where magnetosheath flow is larger than the local Alfvén speed. But because the magnetic field is so strong in this plane the KHI may not grow either. This is also shown in MHD simulations by Chen et al. (1997): in the same scale the tearing mode and Kelvin-Helmholtz cannot operate in the same plane. In the plane perpendicular to this the magnetic fields have anti-parallel components ( because of the draping the $b_{z}$ is negative in MSH and $b_{z}$ is positive in MSP), so that tearing mode may operate in this plane.

Plasma density in the top left panel shows variations at sc1 and sc3 between lower density and higher density regions. During the first $30 \mathrm{~min}$ these encounters occur for about every $10 \mathrm{~min}$ and for latter $30 \mathrm{~min}$ for every $5 \mathrm{~min}$. Plasma velocity oscillations show a more rapid variation. During the first encounter of magnetosheath plasma ( 05:02 UT) the density is not constant but shows variations within this region:sc3 has two encounters with lower density plasma before it returns to the lower density region for a longer time at $\sim 05: 13$ UT. Also, for sc3 the transition back to the lower density region after $05: 10 \mathrm{UT}$ is more gradual than for $\mathrm{sc} 1$.

Throughout this one hour interval, the normal component of the plasma velocity $v_{N}$ shows bipolar and mono-polar variations. The velocity $v_{M}$ typically has the largest values of order $-300 \mathrm{~km} / \mathrm{s}$ in the high density regions which would be characteristic for magnetosheath plasma. The intervals with small positive to zero tangential velocities typically correlate with low density regions and are characteristic for magnetospheric plasma.

Although the variations between magnetosheath and magnetospheric plasma become more frequent after 05:30 UT, a filamentary region of magnetospheric plasma embedded in magnetosheath plasma, at $\sim 05: 19: 30 \mathrm{UT}$, is seen by sc1 and sc3. At this time there is also a rapid change of magnetic field normal component, $b_{N}$, indicative of a very thin current sheet. The $b_{N}$ shows systematic bipolar variations with a periodicity 1-4 min throughout this one hour interval with very rapid variations between 05:15-05:32 UT and longer period in interval 05:40-05:55 UT. At 05:39-05:55 UT the leading edge of the bipolar signature shows a very fast transition whereas the trailing edge shows a smoother transition. The magnetic field $b_{M}$-component shows different types of regions between 05:00-06:00 UT: a negative $b_{M^{-}}$ layer from -20 to $-15 \mathrm{nT}$ corresponding to draped magnetosheath magnetic field along the boundary; a strong positive
$b_{M}$-layer of 20 to $30 \mathrm{nT}$ corresponding to magnetospheric magnetic field; and some intermediate states of -10 to $10 \mathrm{nT}$. The lowest panel on the right-hand side shows the current components: $J_{N}$ (black), $J_{M}$ (red), $J_{L}$ (blue).

The total pressure, $n_{i} k T_{i}+B^{2} /\left(2 \mu_{0}\right)$ (lowest left panel), shows variations typically of $0.2 \mathrm{nPa}$, such that after 05:40 UT the enhanced normal components of $b$ coincide with decreases in the total pressure.

The deHoffmann-Teller frame between 05:00-06:00 UT is rather good with slope of 0.91 and correlation coefficient (cc.) of 0.95 . The corresponding HT-frame velocity in GSM coordinates is $v_{\mathrm{HT}}=[-278,-118,-108]$. In $L M N$-coordinates the HT-velocity is $\sim[-135,-291,-20]$. If we use the $M$ component of the HT-velocity and a $2.5 \mathrm{~min}$ period of bipolar magnetic field oscillations, we can calculate the wave length for this moving structure: $\lambda=v_{\mathrm{HT}_{\mathrm{M}}} *$ $T=291 \mathrm{~km} / \mathrm{s} * 2.2 \mathrm{~min}=6 R_{E}$. This is a huge wavelength compared to spacecraft separation of order $1900 \mathrm{~km}$, which also explains why transitions are so rapid and why different spacecraft encounter these transitions almost (but not quite) simultaneously.

\subsection{Boundary normal oscillations}

Figure 6 shows trajectory of sc1 (moving to positive $z$ direction) between 05:00-06:00 UT with deHoffman Teller frame velocity vectors (red) and boundary normal directions (black) calculated with MFR-method. The top panel has the deHT velocities and boundary normals calculated using $33 \mathrm{~s}$ window and the bottom plot uses a 2 min window. In both cases the window is moved over the data set by approximately $8 \mathrm{~s}$ ( $\sim$ two data points). Both of the boxes are rotated into the average normal (of the one hour interval) direction such that the black vectors show the deviation from the average normal direction. One can see that in both plots the boundary normals oscillate strongly. The 2-min window shows more clearly a quasi-periodic behaviour. The HT-velocity is typically tailward but also earthward directed frames are observed. The most drastic feature is the boundary normal oscillation in $z$-direction which could indicate that the $k$-vector of the waves has a component out from the equatorial plane. We noted earlier that the strong tangential, draped magnetic field along the boundary would stabilise the KHI modes that have $k$-vectors in equatorial plane. However, any mode with $k$-vector out from this plane could grow. Equivalent plots for sc1 and sc4 show similar behaviour (not shown).

In order to test the quality of the normal direction, Fig. 7 shows the details of boundary normal analysis between 05:30-06:00 UT at sc1 (top), sc3 (middle) and sc4 (bottom) (see format in caption).

Because there is an ambiguity of 180 degrees in the normal direction we have assumed that the $y$-component of the normal is always negative (outward pointing normal) and if the method returns a normal with positive $y$, the normal 
components are multiplied by -1 . If the real normal were to have positive values in $y$-direction due to the highly rolled up wave, the change between the outward pointing normal and inward pointing normal measured by one spacecraft would supposedly be gradual and there should be $y$-normal components close to zero before the $y$-component changes sign. One can see in Fig. 7 that the magnitude of the $y$-component measured by sc 1 is smaller than 0.1 in 8 points during this $30 \mathrm{~min}$ interval, so that the normal components following these points could change sign. For sc1 such a region is possibly seen close to $05: 30: 30$ UT where the $y$-component has a small value and in next point $x$ - and $z$-components rapidly change sign. Also at 05:40:10 UT $y$-component has a small value and in next instant $z$-component rapidly changes sign. For many points the normal variation measured by sc1 from one point to the next is reasonably smooth and multiplying these points by -1 would make the changes more abrupt, so that we believe that the oscillations in $z$ - and $x$-components for sc1 are a fair representation of the boundary oscillation during this $30 \mathrm{~min}$ interval. For sc 3 and sc4 there are also points where the $y$-component could become positive, but many of the rapid transitions in $x$ - and $y$-component appear to be real. The angle between the average normal and individual normals show variations between 05:45-06:00 UT with a average period of $\sim 1.2-1.5 \mathrm{~min}$ for sc1, $\mathrm{sc} 3$ and $\mathrm{sc} 4$. We may remind that the bipolar variations of the magnetic field also show an average period of 2 min between 05:4506:00 UT with a faster periodicity of $\sim 1-1.5$ min between 05:17-05:32 UT.

\subsection{Automated search for reconnection intervals}

Figure 8 shows several parameters that are used to identify possible reconnection intervals measured by Cluster spacecraft between 05:30-06:00 UT (see format description in caption). The yellow columns show intervals where the following criteria are simultaneously satisfied: magnitude of Walén relation slope of $0.70-1.1$ and magnitude of correlation coefficient (cc.) $>0.95$, and deHoffmann-Teller frame slope of $0.9-1.1$ and cc. $>0.95$. Sliding (with one data point overlap) window of $33 \mathrm{~s}$ is used for sc1 and sc3, and a $1 \mathrm{~min}$ window for sc4. We note here that the automated search is only a pre-selection for candidate intervals for reconnection so that we must later carefully examine the scatter plots of the Walén relation and HT frames for these intervals.

The average deHoffmann-Teller velocity is about $300 \mathrm{~km} / \mathrm{s}$ which is roughly 75 percent of the solar wind speed. The slopes of the Walén relations show positive and negative values. Paschmann et al. (2005) also found cases with positive and negative slopes of Walén relations during magnetopause crossing of 4th and 5 July 2001. They argue that positive slopes indicate crossings sunward and negative slopes tailward of an X-line, respectively. One can also notice intervals with accelerated and decelerated deHoffmann-Teller frames. For all spacecraft this accelerations/decelerations typically

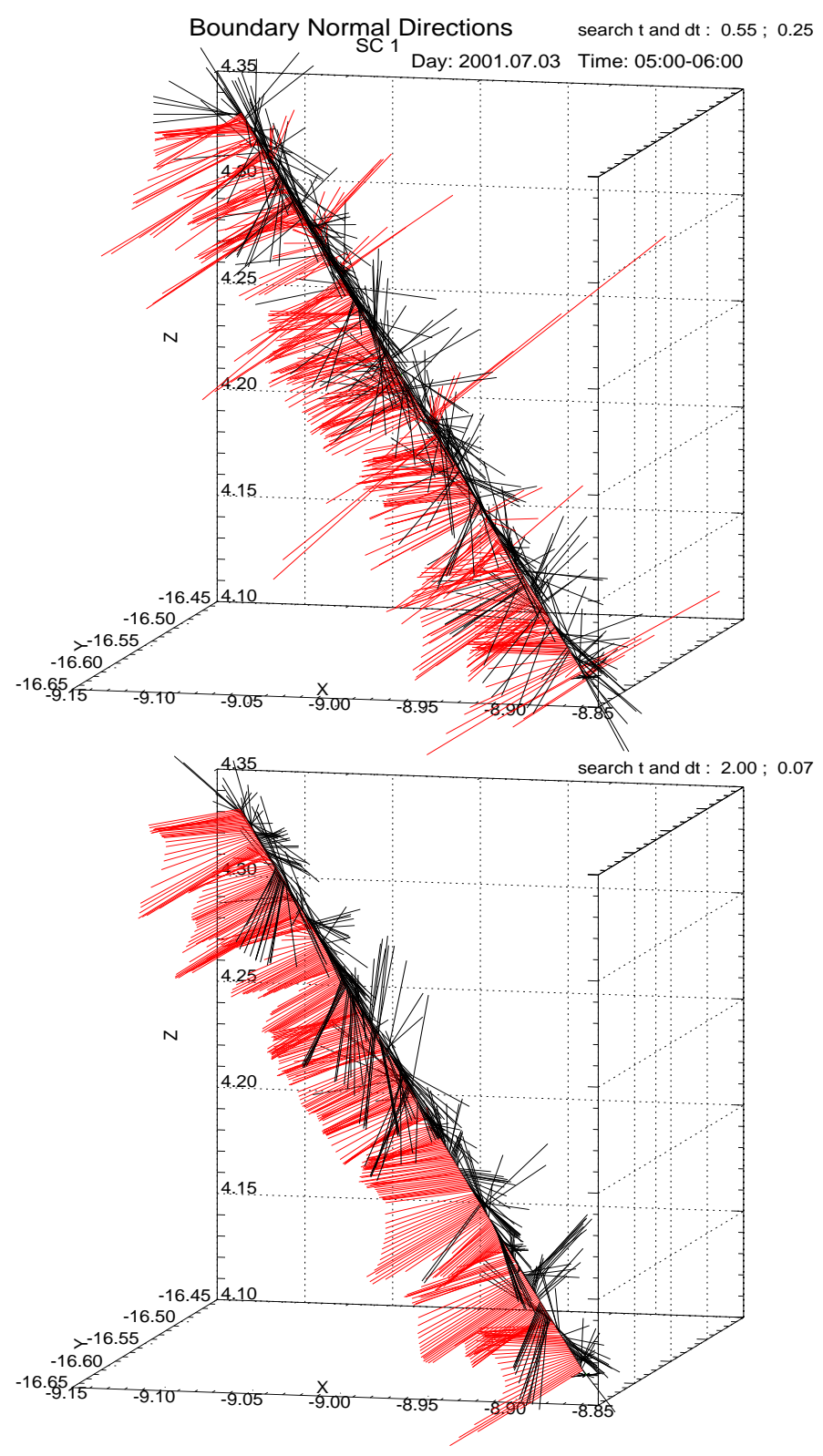

Fig. 6. Sc1 observations between 05:00-06:00 UT of the boundary normal vectors (black) and deHoffman Teller frame vectors (red) along spacecraft trajectory when using $0.55 \mathrm{~min}$ nested intervals (top) and $2 \mathrm{~min}$ nested intervals (bottom). The view is along the average normal direction, such that the black vectors indicate a deviation from the average normal. Equivalent plots for sc3 and sc4 show similar type of behaviour of the boundary normals and deHoffman Teller frame vectors.

occur in the region of lower Alfvén Mach number (Alfvén Mach number is shown for sc1 and sc3 and sc4 in the third panel), which typically should be the case for reconnection: the reconnection is usually switched off if the plasma flow velocity is larger than the local Alfvén speed. 

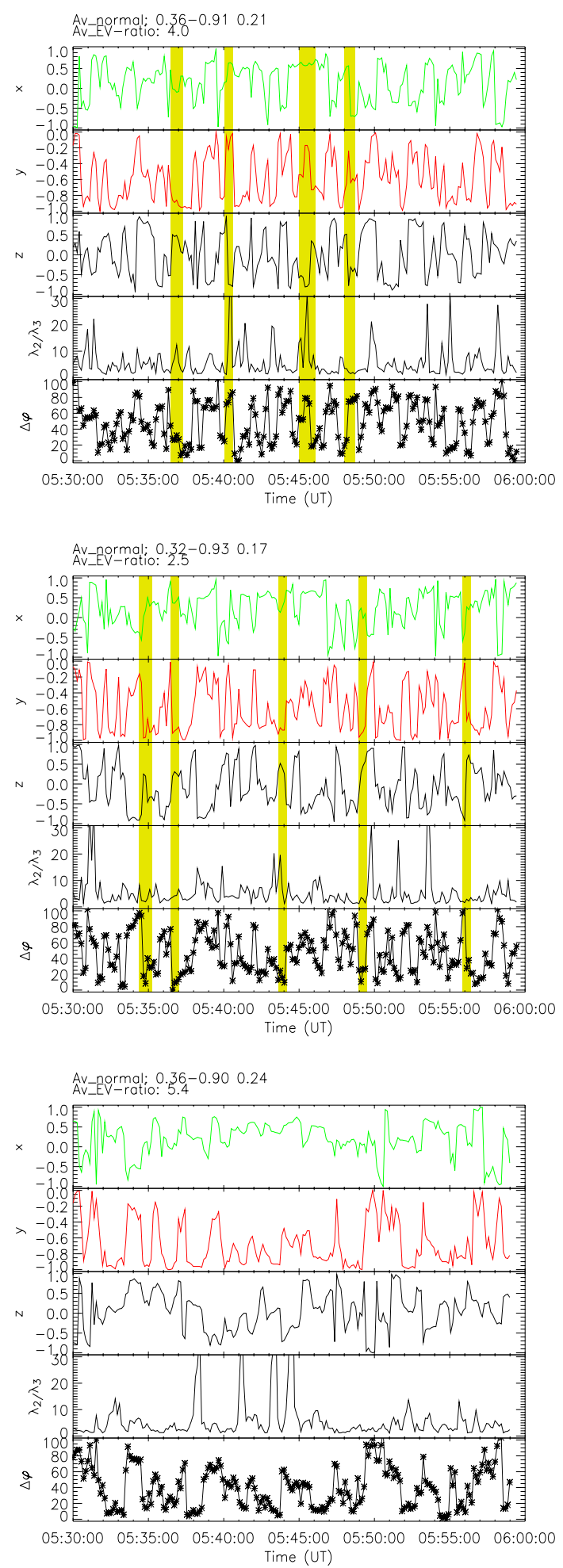

Fig. 7. Sc1 (top), sc3 (middle) and sc4 (bottom) observations between 05:30-06:00 UT of the $x$ - (green), $y$ - (red) and $z$ - (black) components of the boundary normals calculated with MFR -method (top panel), eigenvalue ratios $\left(\lambda_{2} / \lambda_{3}\right)$ of the MFR-analysis (middle panel), angle between average normal and individual normal (bottom panel). The normal calculated using $30 \mathrm{~min}$ of data for sc1 is [0.36,-0.91,0.21] (in GSM) with eigenvalue ratio of 4.0 and is very similar for the other 2 spacecraft (marked in upper left corner in each plot). It is pointing mostly toward the negative $y$-direction, which is consistent with the spacecraft crossing the dawnside magnetopause. The yellow columns show intervals where the reconnection criteria (explained later) is satisfied. 

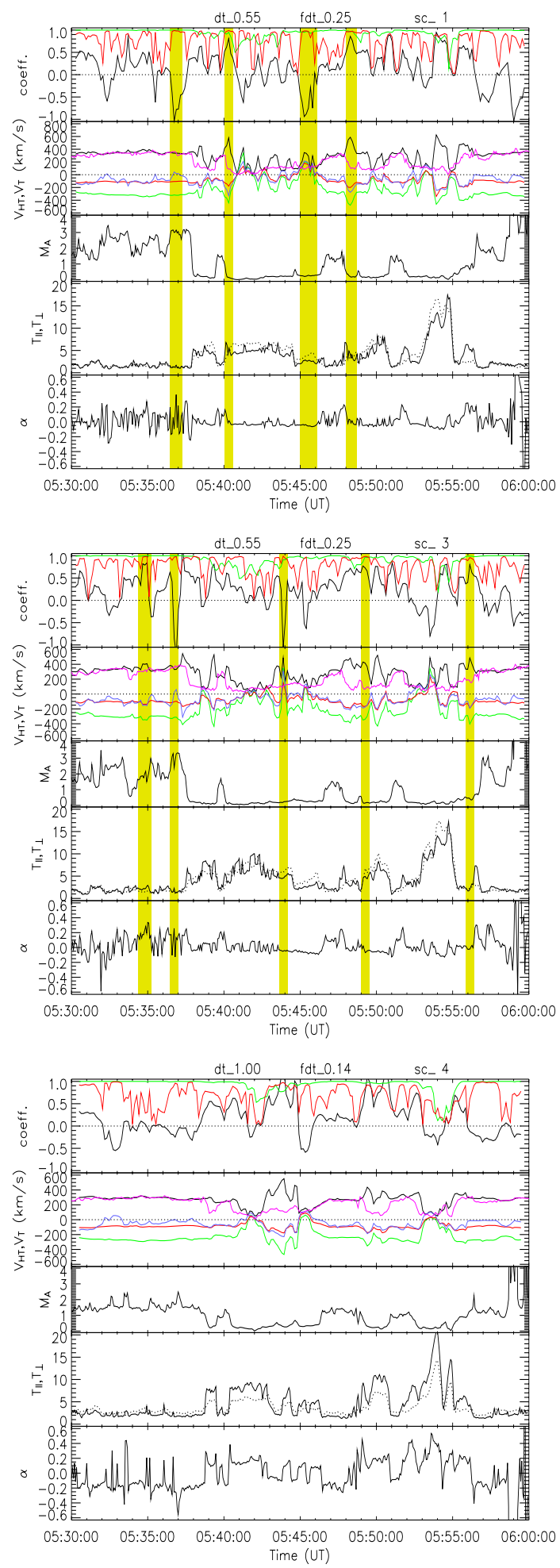

Fig. 8. Sc1 (top), sc3 (middle) and sc4 (bottom) observations of various plasma parameters between 05:00-06:00 UT. Top panel shows observations of the slopes of the Walén relation (black), slopes of the deHoffmann-Teller frame (green) and magntitude of correlation coefficient of the Walén relation (red). The second panel shows components of the deHoffmann-Teller frame velocity: $v h t_{t}$ (black), $v h t_{x}$ (green), vht $t_{y}$ (red), $v h t_{z}$ (blue) and total plasma velocity $v_{t}$ (magenta). Third panel shows the Alfvén Mach number, the fourth panel shows parallel (solid line) and perpendicular(dotted line) temperature and the fifth panel shows the value of the anisotropy factor $\alpha$. 
Between 05:30-06:00 UT sc1 observes 4 candidate intervals (a thicker yellow column indicates that there are several nested $33 \mathrm{~s}$ intervals within the column) for reconnection. Sc3, which is furthest equatoward, observes 5 candidate intervals for reconnection and sc 4 doesn't observe any intervals (probably due to the longer interval of calculation and time resolution). Two of the intervals for sc1 and sc3 have negative slopes of Walén relation, and positive slopes are observed during 2 intervals for sc 1 and during 3 intervals for sc3. We will next remove intervals, that satisfy the search criteria but that have the data points clustered along the diagonal in Walén relation or in HT frame. A detailed examination of the $33 \mathrm{~s}$ intervals marked with yellow in Fig. 8 confirms that only two (intervals starting at $\approx 05: 40$ UT and 05:48 UT) of the sc1 intervals are good, and only the last two of the sc 3 intervals are good. It is also interesting that the total plasma velocity slightly decreases during the intervals when reconnection condition is satisfied well for sc 1 and sc3. This is possible if the tension from the reconnected field lines is in the opposite sense than the magnetosheath flow speed. We will return to this in next section when discussing the simulation results.

Using 1-min intervals for sc1 and sc3 with the same criteria yields one interval for both spacecraft satisfying the reconnection criteria. However, a closer examination of sc1 interval at 05:36:30-05:37:30 UT indicates that the HT-frame components are clustered along the diagonal. For sc3 a detailed examination of Walén relation and HTframe at 05:48:52-05:49:52 UT show a good HT-frame with $v_{\mathrm{HT}}=[-358,-174,-189]$, slope $=0.95$, and a Walén relation with slope of 0.80 and $c c .=0.98$. Clusters along diagonal could arise from the partial crossing of the rotational discontinuity. That is, if the interval corresponds to the crossing of the partial boundary layer, then the HT frame would be good and accelerated/decelerated, and the flows would be Alfvénic. However, because the interval does not include the rotation of the field, the components of the local Alfvén velocity don't change much and form "clusters" along the diagonal. Also, the Walén test is applicable only to plasma on connected field lines, otherwise the test will give meaningless results.

Figure 9 represents Walén relation (left) and deHoffmannTeller frame (right) measured by sc1 between 05:48:0005:48:33 UT (top), sc3 between 05:48:58-05:49:31 UT (middle), and sc4 between 05:48:54-05:49:34 UT (bottom). The slopes of Walén relation are $0.86,0.73$ and 0.99 for sc1, $\mathrm{sc} 3$ and sc4, respectively. The corresponding correlation coefficients are $0.98,0.98$ and 0.96 such that sc 4 shows a larger variance in the spread of the points. We note that this sc4 interval is not picked in the automated test (shown in Fig. 8) because the 1-min interval does not satisfy the search criteria for sc4. Here we have used only 6 data points for sc 4 in order to compare with sc1 and sc 3 results. The deHoffmann-Teller frames are good for all 3 spacecraft and HT-velocities are pointing tailward, southward and dawnward. Sc1 measures the highest HT-velocity of $v_{\mathrm{HT}}=[-479,-201,-276]$, and sc3 and sc4 measure roughly $100 \mathrm{~km} / \mathrm{s}$ lower HT-velocities. All of these HT-frame velocities are higher than that of the overall structure between 05:30-06:00 UT, for which sc1 measures HT-velocity of $v_{\mathrm{HT}}=[-292,-122,-115]$. We interpret that sc3 and sc4 are encountering the same reconnection layer - although crossing it slightly differently.

The fact that reconnection is observed by only one or two of the spacecraft simultaneously indicates that while the reconnection region may be as long as 1 to $2 R_{E}$ they certainly must be rather thin $(<1000 \mathrm{~km})$ and for the identified cases be aligned mostly tangential to the boundary flow otherwise all spacecraft should see them. This, however, may be only a subset of all reconnection events because if they cross the spacecraft at a steeper angle the crossing would only take a few seconds and they wouldn't be identified.

Finally, we note that the Walén test is very sensitive to plasma number density measurements. Inaccuracies in this measurement would result in a change of the slope while the correlation coefficient would remain roughly unchanged. HIA instrument tends to underestimate the number densities in the magnetosphere, and CODIF instrument tends to underestimate number densities in the magnetosheath. This will tend to underestimate the slopes of the Walén relation for the HIA intervals, and will tend to overestimate the slopes for the CODIF intervals. So perhaps more HIA intervals would satisfy the search criteria for reconnection. Also, in the Walén test we have here only considered protons, so in the presence of the heavier ions this test would fail or get worse.

\section{MHD simulation of KHI with the initial conditions from Cluster observations}

Results in this section are obtained with 2.5-dimensional MHD simulations (Otto, 1990; Nykyri, 2002). The basic equations are solved with a finite differences leapfrog scheme (Potter, 1973; Birn, 1980), which is of second-order accuracy in space and time.

The density, pressure, velocity, and magnetic field on the magnetospheric and magnetosheath sides at the initial shear flow plane are chosen according to Cluster observations of 3 July 2001 between 05:00-06:00 UT.

The initial state of the simulation uses a Galilei transformation for the velocity such that the simulation frame is moving with half the magnetosheath velocity. In this manner the $\mathrm{KH}$ vortex is moving slower in the simulation frame, with the advantage that the time step limit for the integration of the MHD equation is larger than in a frame which is at rest with the magnetosphere. Boundary conditions for the simulations are periodic along the $x$-direction, and boundary conditions in $y$ are open to inflow and outflow of plasma.

The values for the normalization of the simulation units are summarized in Table 1. All quantities are normalized to characteristic values for the system, i.e., length scales $l$ to a 

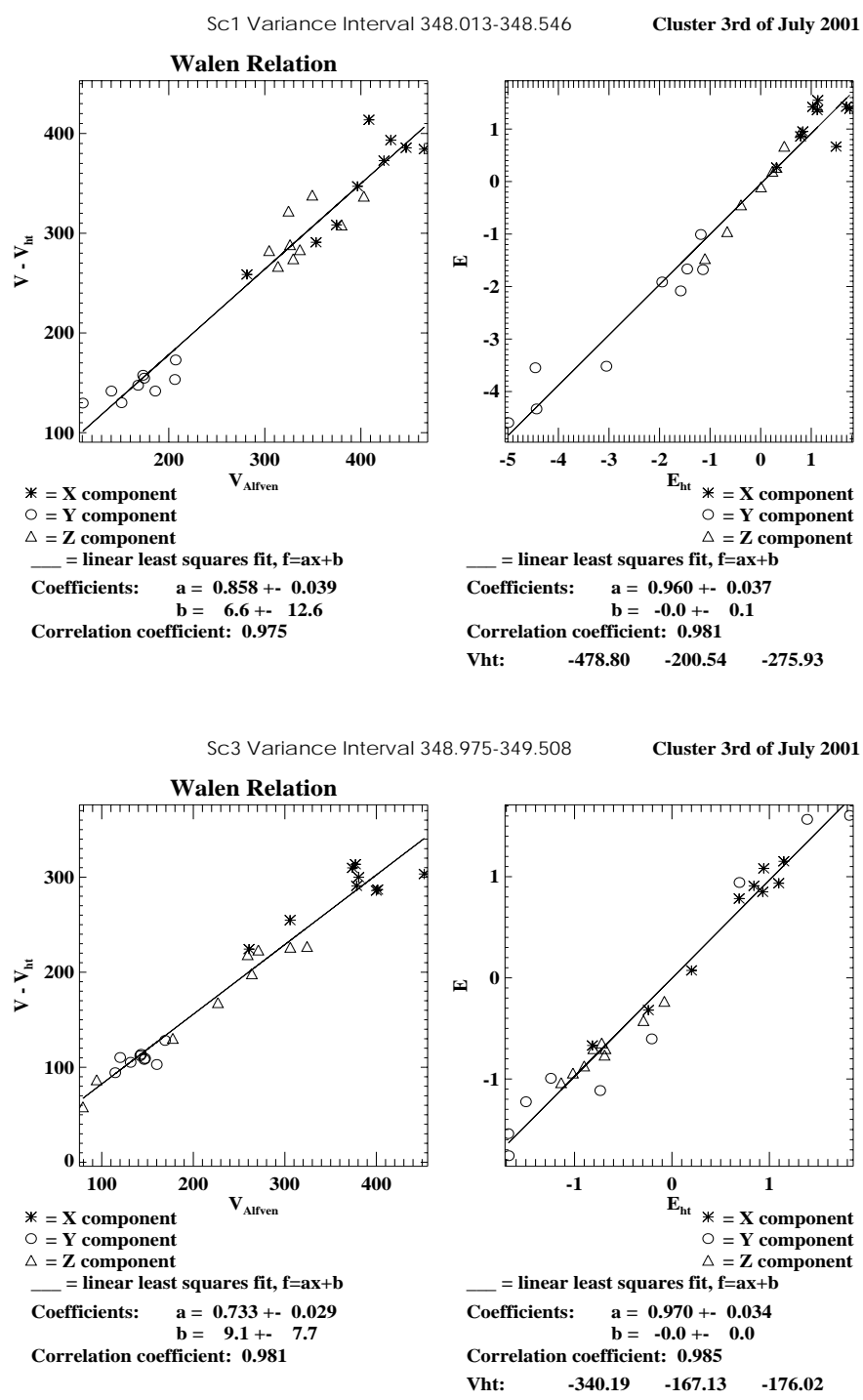

Sc 4 Va riance Interval 348.900-349.567 Cluster 3rd of July 2001
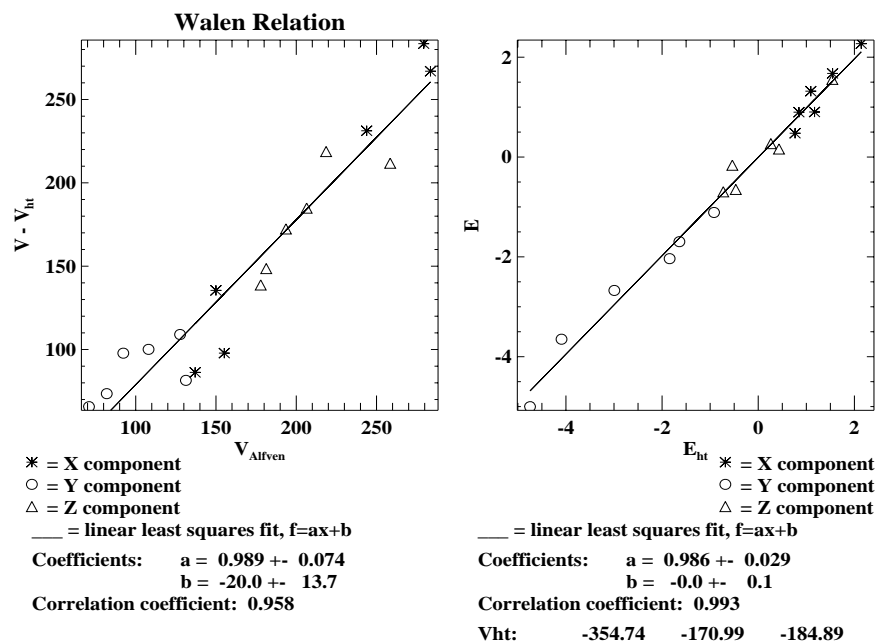

Fig. 9. Walén relation (left) and deHoffmann-Teller frame (right) measured by sc1 between 05:48:00-05:48:33 UT (top) sc3 between 05:48:58-05:49:31 UT (middle) and sc4 between 05:48:54-05:49:34 UT (bottom). 
Table 1. Simulation normalization.

\begin{tabular}{lr}
\hline Magnetic field $B_{0}$ & $27 \mathrm{nT}$ \\
Number density $n_{0}$ & $7 \mathrm{~cm}^{-3}$ \\
pressure $p_{0}$ & $0.29 \mathrm{nPa}$ \\
Current density & $22 \mathrm{nA} \mathrm{m}^{-2}$ \\
Length scale $L_{0}$ & $1000 \mathrm{~km}$ \\
Velocity $v_{A}$ & $222 \mathrm{~km} \mathrm{~s}^{-1}$ \\
Time $\tau_{A}$ & $4.5 \mathrm{~s}$ \\
\hline
\end{tabular}

typical length $L_{0}$; density $\rho$ to $\rho_{0}=n_{0} m_{0}$ with the number density $n_{0}$ and the ion mass $m_{0}$; magnetic field $\boldsymbol{b}$ to $B_{0}$; velocity $\boldsymbol{v}$ to the typical Alfvén velocity $v_{A}=B_{0} / \sqrt{\mu_{0} \rho_{0}}$; pressure to $P_{0}=B_{0}^{2} /\left(2 \mu_{0}\right)$; current density to $J_{0}=B_{0} /\left(\mu_{0} L_{0}\right)$; and time $t$ to a characteristic Alfvén transit time $\tau_{A}=L_{0} / v_{A}$. The system size is 40 units in $x$, corresponding to a wavelength of about $6 R_{E}$. The simulation employs $549 \times 429$ gridpoints in the $x$ - and $y$-directions.

Figure 10 shows simulation geometry. In the middle panel the black lines represent Earth's magnetic field lines and red lines indicate the draped IMF for Parker spiral configuration. The initial shear flow plane is constructed by using the typical observed magnetic field orientations between 05:0006:00 UT on magnetospheric and magnetosheath sides of the boundary which are projected along the observed sheath velocity. In this plane the magnetospheric and magnetosheath fields are in $\sim 165$ degree angle, such that $\alpha \sim 68$ and $\beta \sim 53$. In the shear flow plane the magnetic fields are anti-parallel, such that the magnetospheric field is $\sim 25 \mathrm{nT}$ and orientated anti-parallel to sheath flow, and the magnetosheath field is $\sim-19$ nT pointing along the sheath flow. The magnetic fields perpendicular to shear flow plane (positive z-direction) are $\sim 10 \mathrm{nT}$ and $\sim-14 \mathrm{nT}$ in magnetospheric and magnetosheath side respectively.

Simulations in this geometry (not shown) indicate that the growth of KHI is stabilized by the strong tangential magnetic field because $\Delta \boldsymbol{v}_{s h} \cdot \boldsymbol{k}=2<\Delta \boldsymbol{v}_{A} \cdot \boldsymbol{k}=2.91$.

The observations showed a strong variation of the $z$ component of the boundary normal. Also the deHoffmannTeller frame velocities and the actual plasma velocity had a significant $z$-component. These observations indicate a possibility of a KH mode with $k$-vector out from the equatorial plane. Figure 11 shows simulation result at $t=91.32=410 \mathrm{~s}$ in the plane that is projected along the $k$-vector that is tilted by -35 degrees from the initial shear flow plane. This corresponds to a counter-clockwise rotation about the $y$-axis of the initial shear flow plane such that the velocity shear across this plane is reduced to $v_{s h} \cos (35)$, but also the average tangential magnetic field along the $k$-vector is reduced so that $\Delta \boldsymbol{v}_{s h} \cdot \boldsymbol{k}=1.64>\Delta \boldsymbol{v}_{A} \cdot \boldsymbol{k}=1.6$.

In Fig. 11 the magnetic field (arrows) and z-component of the current density, $j_{z}$ (color coded), are plotted on the top left panel, and velocity (arrows) and density (color coded) on top right panel. The black lines are magnetic field lines projected on the shear flow plane. The wave is moving into negative $x$-direction (down the page) and the higher density side indicates the magnetosheath side. One can see a nonlinear KH wave with two oppositely directed elongated thin current sheets adjacent to each other with ongoing reconnection at $x, y \sim[0 .-7]$. The MHD code uses current dependent resistivity, such that resistivity is switched on when threshold current of $1.1 J_{0}$ is exceeded.

The reconnection has first occurred in the layer of negative current between anti-parallel magnetosheath and magnetospheric fields and has generated two oppositely moving flux tubes. The other reconnected flux tube is moving downstream with the wave and other one is connected upstream. In these flux tubes both magnetospheric and magnetosheath plasma are present.

The second reconnection occurs in the layer of positive current in the low density region between twisted magnetospheric fields. Reconnection has generated a magnetic island moving downstream, and the upstream moving flux tube has both ends connected to the Earth's magnetic field, so that these flux tubes only have magnetospheric plasma in them.

We note that the KH dynamics for this magnetic field configuration is different from the dynamics of $\mathrm{KH}$ evolution with initially strongly parallel magnetic fields (Nykyri and Otto, 2001). In this case KHI twists the parallel fields and generates reconnection in the high density part of the vortex. Here the initially anti-parallel fields are made parallel by the $\mathrm{KHI}$ in the high density part of the vortex, preventing reconnection at that location.

The bottom left panel has a cut through at $y=2$ at magnetospheric rest frame, corresponding to measurements of a virtual spacecraft as the wave passes by it. The bottom right panel has Cluster sc1 and sc3 data between 05:46:3005:51 UT in boundary normal coordinates of sc1. In both plots the magenta colored columns represent intervals where good Walén relations were observed. The boundary normal and tangent used in this plot are calculated with MFR method between 05:42-05:54 UT from sc1 data. The normal is $[0.49,-0.86,-0.1]$, and the tangent is $[0.49,0.37,-0.78]$. In both plots the top panel shows plasma number density (solid line) and plasma temperature (dashed line). Second panel shows total pressure, third panel has normal (solid) and tangential (dashed) component of plasma velocity, and fourth panel shows normal (solid) and tangential (dashed) component of the magnetic field. In simulation data we have multiplied the normal $(y)$-direction with -1 and rotated the simulation data with -10 degrees about the $z$-axis in order to better compare with $N L$-coordinates of the data. Although this is a two-dimensional simulation result, many features qualitatively agree with Cluster observations. Both plots show similar type of variation of sheath-like (high density, low temperature) and magnetospheric-like (low density high temperature) regions, and the location of the intervals satisfying the reconnection criteria are also in good 


\section{$\underline{\text { Kelvin-Helmholtz Simulations }}$}
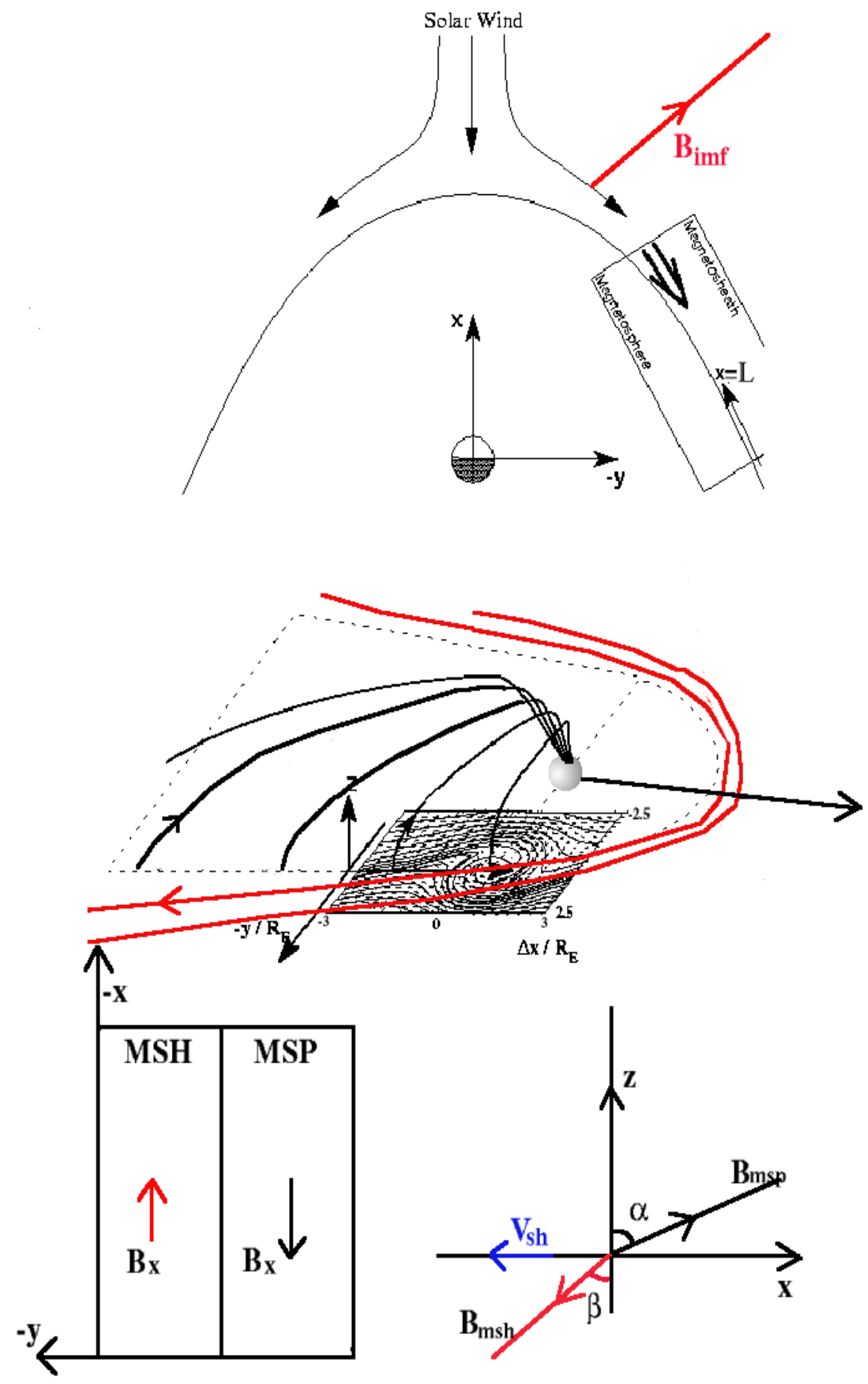

Fig. 10. Simulation geometry.

agreement. The minimum total pressure is obtained at the center of the vortex (not shown), but here the cut is at the magnetospheric side of the wave, so that the pressure vari- ation is smoother. The normal component of the magnetic field and velocity show a clear signature in the simulation data between $x=[1,4]$ by changing from $3 \mathrm{nT}$ to $-15 \mathrm{nT}$ and 

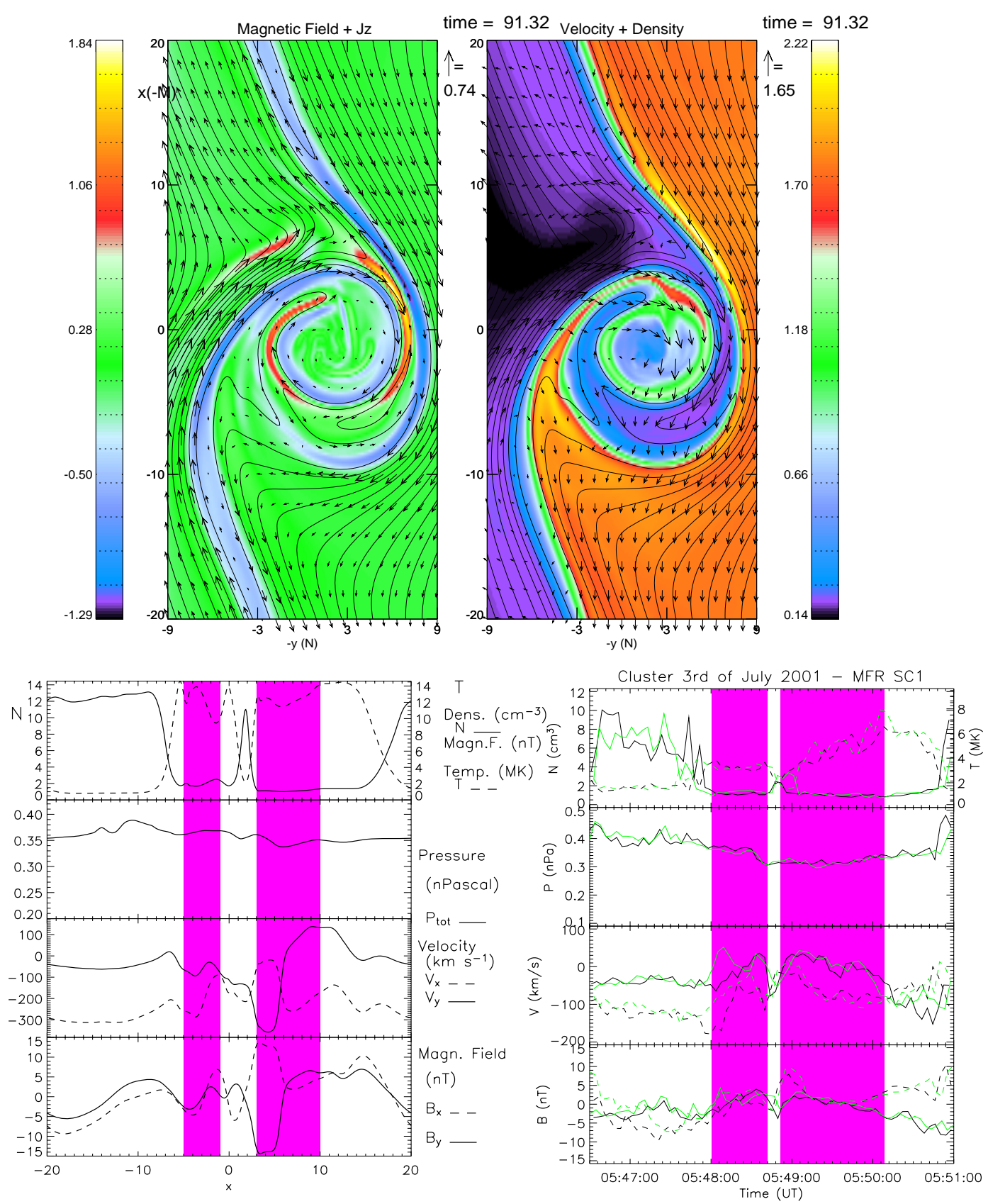

Data represents a cut throuth system for const $y$

Location is in simulation units and velocities are in magnetospheric frame

Cut at $y=2.0$, phi $=0.0$, time $=410$.

Fig. 11. MHD simulation result of the KH wave at $t=410 \mathrm{~s}$. The magnetic field and $j_{z}$ are plotted on the top left panel and velocity and density at top right panel. The black lines are magnetic field lines. The bottom left panel has a cut through at $y=2$, corresponding to a measurements of a virtual spacecraft as the wave passes through it. The bottom right panel has Cluster sc1 and sc3 data between 05:46:30-05:51 UT in boundary normal coordinates of sc1. The data panels show from top to bottom plasma density and temperature, total pressure, normal (solid line) and tangential (dashed) component of plasma velocity, normal and tangential component of the magnetic field. The intervals satisfying reconnection criteria are marked with vertical magenta columns.

from $-120 \mathrm{~km} / \mathrm{s}$ to $-350 \mathrm{~km} / \mathrm{s}$, respectively. In Cluster data this signature is present between 05:48:36-05:48:48 UT, but the magnitudes are off: $v_{N}$ drops from $40 \mathrm{~km} / \mathrm{s}$ to $-70 \mathrm{~km} / \mathrm{s}$ and $b_{N}$ from $4 \mathrm{nT}$ to $-4 \mathrm{nT}$. This signature occurs in both plots in the vicinity of the higher density filament, although in Cluster data the magnitude of this density filament is three 

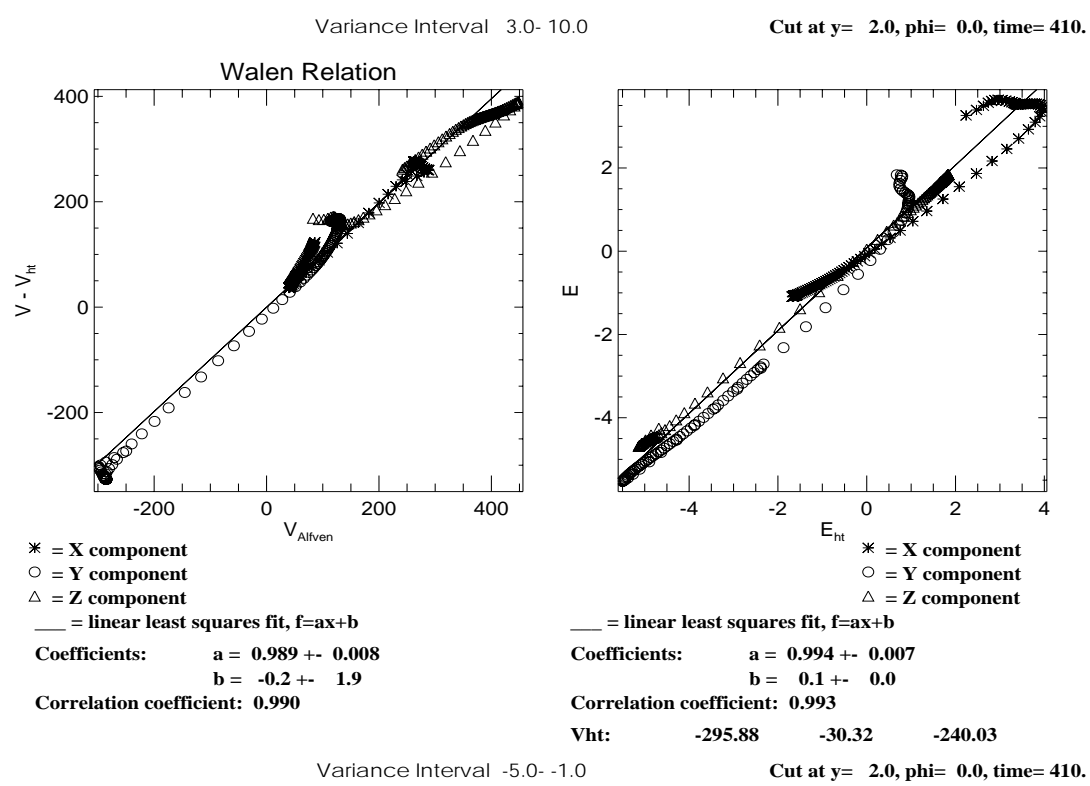

Variance Interval $-5.0--1.0$

Cut at $\mathbf{y}=\mathbf{2 . 0}, \mathrm{phi}=\mathbf{0 . 0}$, time $=410$.
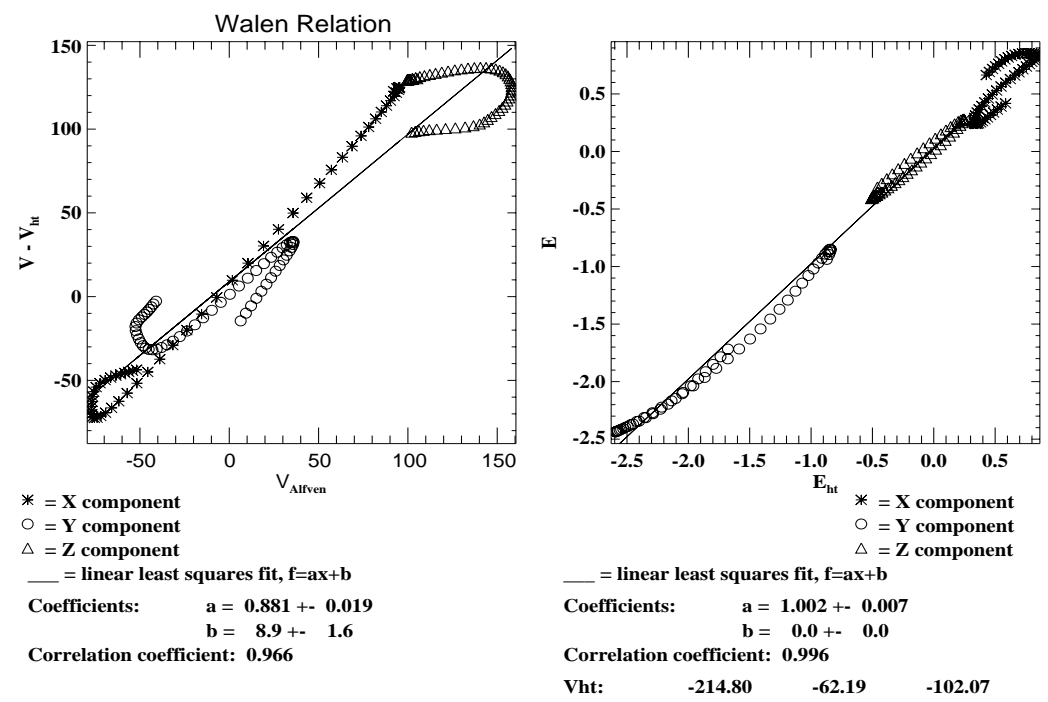

Fig. 12. Walén relation (left) and deHoffmann-Teller frame (right) "measured" by virtual spacecraft during cut at $y=2$ between $x=[3,10]$ (top), and between $x=[-5,-1]$ (bottom).

times lower than in the simulation. Based on the simulation such a filament and normal components are arising due to the rolled up nature of the Kelvin-Helmholtz wave. Signature is reversed between $x=[5,9]$ when $v_{N}$ and $b_{N}$ change from $-350 \mathrm{~km} / \mathrm{s}$ to $130 \mathrm{~km} / \mathrm{s}$ and from $-15 \mathrm{nT}$ to $7 \mathrm{nT}$, respectively. In data the corresponding transition is again smoother and $v_{N}$ and $b_{N}$ change back to their original values. There are also slight differences between $\mathrm{sc} 1$ and $\mathrm{sc} 3$ observations, because sc1 locates earthward (in $x, y$-plane) of sc3 and it is also located $\approx 1200 \mathrm{~km}$ in $+z$-direction from sc3. In context of top left panel of Fig. 5 we argued that the continuous chain of bipolar variations of the normal component (with rapid leading edge and smoother trailing edge) is indicative of a wave. A smooth trailing edge of the $b_{N}$ can be seen be- tween 05:49:06-05:51 UT (in Fig. 5 and in Fig. 11), where $b_{N}$ changes from $4 \mathrm{nT}$ to $-8 \mathrm{nT}$. This signature is also clearly present in the simulation data between $x=[9,20]$, which supports our argument on the origin of the systematic bipolar variations of the $b_{N}$. Also in favor of the KHI, the the total pressure depression occurs between $x=$ [5.5-7], during interval when normal component of the magnetic field increases to its maximum and the Walén relation is well satisfied. In case of a FTE, a total pressure maximum should occur (Paschmann et al., 1982). Also, the tangential components show similarities between data and observations although the magnitudes in magnetosheath side (high density side) are slightly off. This is because we have normalized the simulation velocities into a Alfvén speed that uses the 


\section{CIS-HIA RUMBA (SC 1) 03/Jul/2001 05:47:55.601}
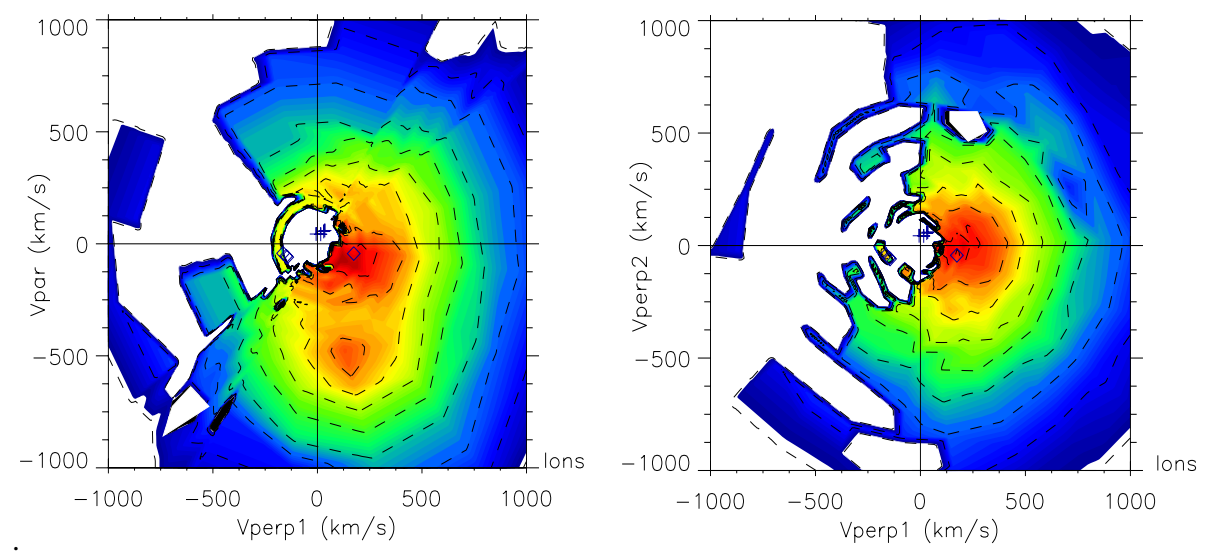

Fig. 13. The left panel shows ion distribution function at parallel/perpendicular-plane (right panel has perpendicular cut) from HIA instrument onboard sc1 at 05:47:55 UT.

average density of the magnetosheath and magnetospheric side $\left(7 / \mathrm{cm}^{3}\right)$ and magnetospheric magnetic field of $(27 \mathrm{nT})$. Therefore the Alfvén speed and thus velocity in the magnetosheath side is higher by factor of $\approx 1.6$.

It is now interesting to consider virtual spacecraft observations of the discontinuities generated in the simulation box by the KHI. Figure 12 has the same format as Fig. 9, showing the Walén relations and HT-frames at different locations in the simulation box at $t=410 \mathrm{~s}$ in magnetospheric rest frame.

The top panel of Fig. 12 shows Walén relation (slope $=0.99$ and $c c .=0.99$ ) and HT frame (slope $=0.99$ and $c c .=0.99$ ) during a cut at $y=2.0, x=[3 ., 10$.$] . This corresponds to a cross-$ ing of a rotational discontinuity at the magnetospheric (low density) side. This interval is marked with second magenta colored column in Fig. 11 and corresponds to sc3 interval in Fig. 9. The HT frame velocity $\left(\boldsymbol{v}_{\boldsymbol{H} T}=[-296,-30,-240]\right)$ has accelerated in $-z$-direction (the HT frame velocity for the entire wavelength (not shown) between $x=[-20,20]$ is $[-300,-47,-119])$. The Walén relation slope has same sign as in Fig. 9 and it is positive because at the location of the crossing the $v_{N}$ and $b_{N}$ have same signs, which can be confirmed in data and simulation comparison in Fig. 11. The X-line is located tailward and duskward from the location of this rotational discontinuity. One can also see that the field line tension of these flux tubes is directed in the opposite direction than the magnetosheath flow speed, consistent with observed decrease in total velocity in Fig. 8. Sc4 locates $\approx 1600 \mathrm{~km}$ along normal direction from sc3. The bottom panel of Fig. 9 indicates that (if we can trust 5 data points) it observes similar result consistent with $\mathrm{sc} 3$ data. The corresponding cut in simulation box (not shown) at $y=0.4(1.6 \times 1000 \mathrm{~km}$ separation from $\mathrm{sc} 3)$ between $x=[4,7]$ shows excellent Walén relation (slope $=1.0$ and cc. $=1.0$ ) and HT frame (slope $=0.98$ and $c c .=0.99$ ) with $V_{\mathrm{HT}}=[-284,-37,-222]$.
The bottom panel of Fig. 12 shows the Walén relation (slope $=0.88$ and $c c .=0.97$ ) and HT frame (slope $=1.0$ and cc. $=1.0)$ for a cut at $y=2, x=[-5,-1]$. This corresponds to a crossing of a reconnected flux tube, that originates from the layer of negative current. Due to the twisting of the boundary by KHI the magnetic field geometry is here more complex than in Fig. 3 by Paschmann et al. (2005). The Walén slope is here positive although the $\mathrm{X}$-line is sunward and duskward from the reconnected flux tube. Here also, the tension of the reconnected flux tube is oppositely orientated from the average magnetosheath flow, consistent with observed decrease in total velocity in Fig. 8. This flux tube shows a HT-frame velocity of $\boldsymbol{v}_{\mathbf{H T}}=[-214,-62,-102]$. Although the location and timing of this interval matches the observed reconnection interval by sc1 (the first magenta column in Fig. 11), the HT frame velocity is much lower than that of the first panel of Fig. 9. The reason for this can be seen in Fig. 13. The left panel shows an ion distribution function in the parallel/perpendicular plane from the HIA instrument onboard sc1 at 05:47:55 UT. Sc1 detects a main magnetosheath-type distribution function together with an additional field-aligned ion beam with $v_{\|}=500 \mathrm{~km} / \mathrm{s}$. Moment calculations of such a distribution function are affected by the existence of such multiple populations, which in turn affects the HT frame velocities, i.e., which will be larger here. Such a distribution function (not shown) is also observed by sc1 at $\sim 05: 40$ UT and by sc 3 at $\sim 05: 56 \mathrm{UT}$, during intervals when reconnection criteria was met. One can see in Fig. 8 that during these three intervals the plasma parameters and coefficients are very similar. Because such a double population is observed at three different times, and during at least two successive distribution functions (separated by $12 \mathrm{~s}$ ), their co-existence is likely real rather than due to a time-aliasing effect. These beams could be particle signatures of reconnection. In MHD simulations such kinetic effects are not present. 

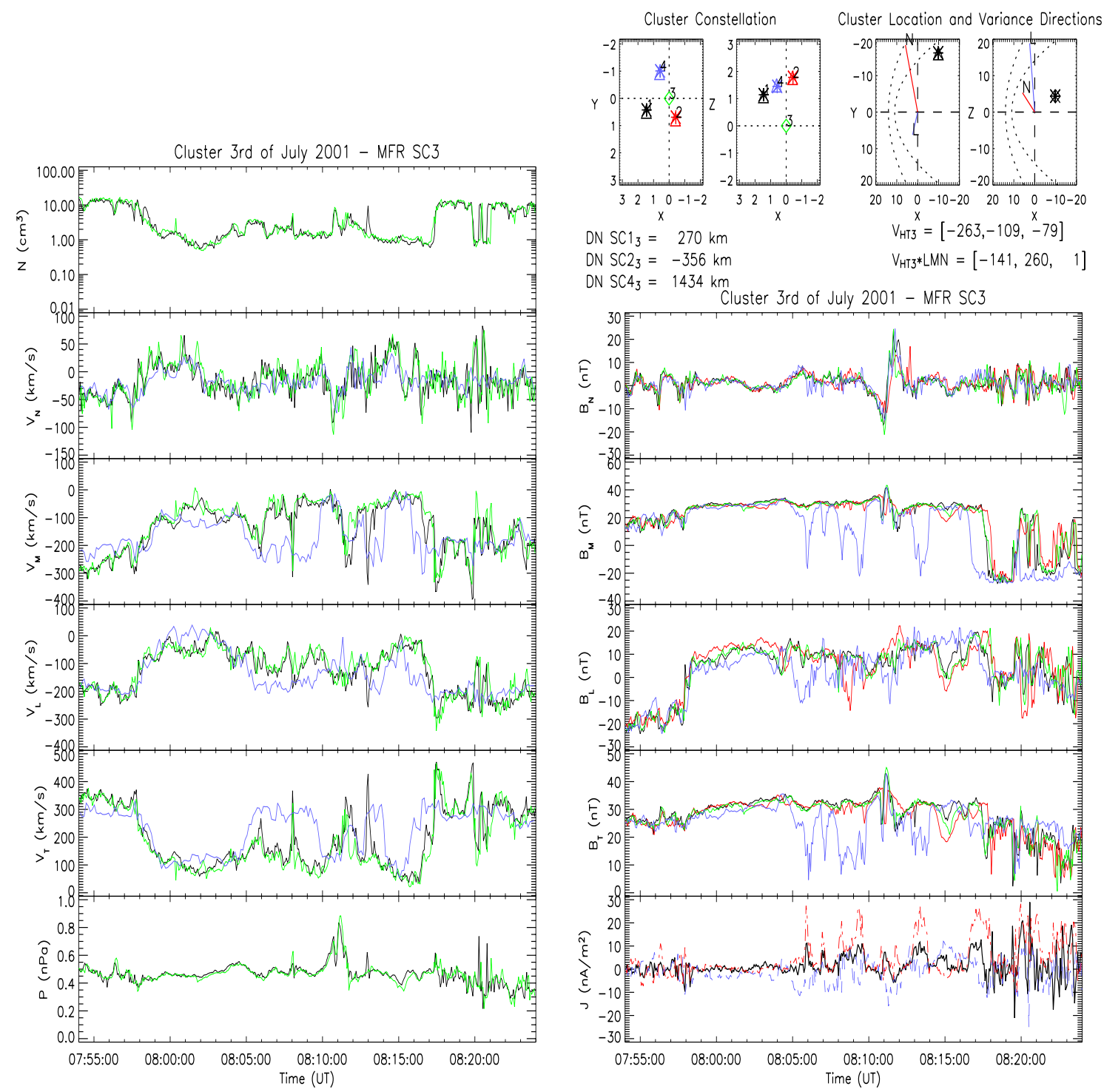

Fig. 14. Plasma (left) and magnetic field (right) observations between 07:54-08:24 UT in the boundary normal coordinates of sc3. Figure uses same format as Fig. 5.

The automated search in Fig. 8 returned intervals when negative slopes of the Walén relation were observed, but the closer examination of these showed that the points were slightly clustered along diagonal. In the simulation box the negative slopes can be found when crossing the boundary between not mutually connected fieldlines from low density to intermediate density: at simulation time $t=91.32$ (top panel of Fig. 11) the cut at $y=3.2, x=[16 ., 19$.$] , gives a slope of$ -0.49 . Cluster sc1 observed an interval with negative Walén slope at 05:45 UT and sc3 at 05:44 UT. If we take the same cut in the simulation box slightly earlier at $t=88.66$, corresponding to a time when reconnection has first occurred at the layer of negative current, the Walén slope is -0.9 . In this case the reconnection has not yet occurred in the layer of positive current. However, in both of these cases both the Walén relation and HT-frame look slightly clustered. During the observations of negative slopes, the Cluster spacecraft could be crossing a boundary between magnetic field lines where the other flux tube is originating from X-line at the layer of negative current and other from layer of positive current. Because of the periodic boundary conditions of the simulation box one can either say that these $\mathrm{X}$-lines and current layers are either tailward or earthward of the spacecraft. Same should also apply for the real magnetopause if Kelvin-Helmholtz wave trains travel along it. 

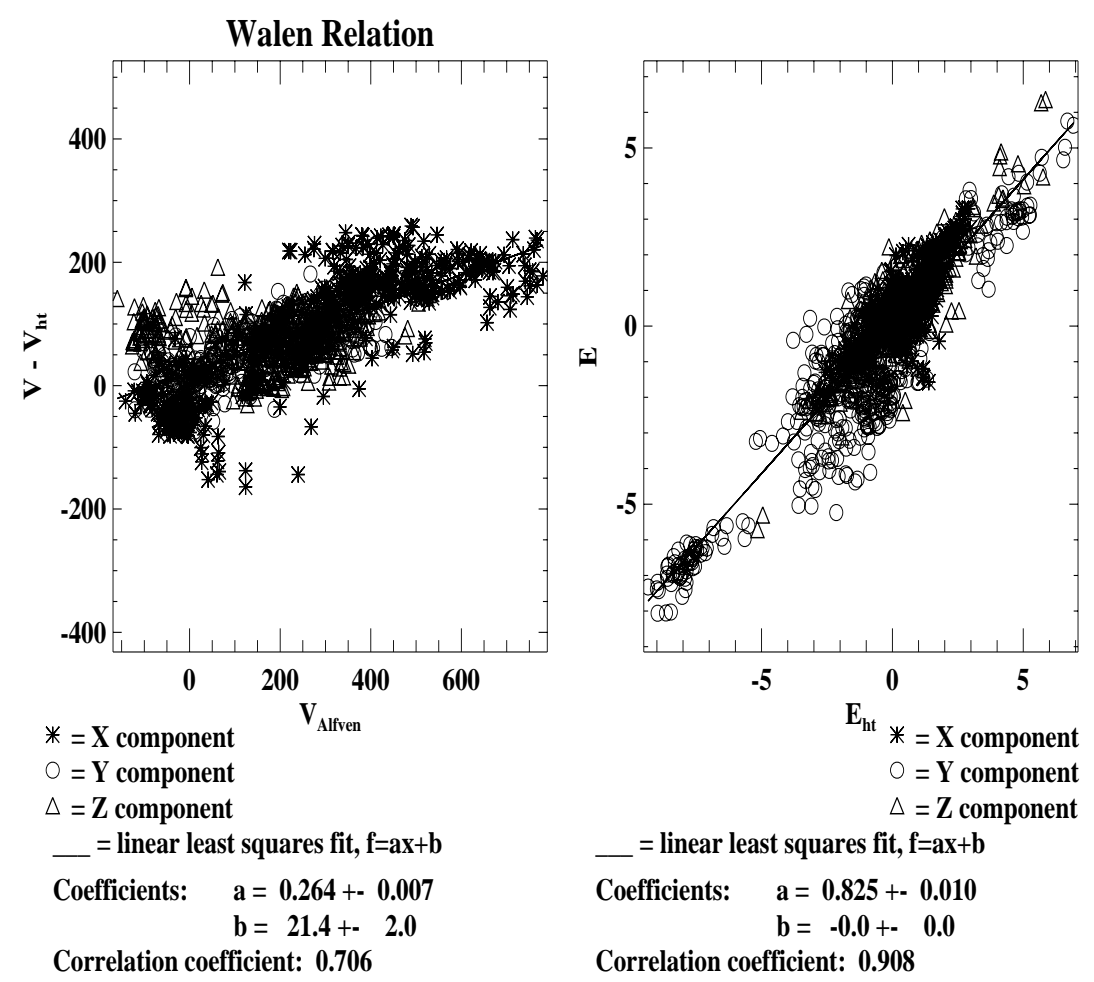

$\ldots$ _ linear least squares fit, $\mathrm{f}=\mathrm{ax}+\mathrm{b}$

Coefficients: $\quad a=0.825+-0.010$

Correlation coefficient: 0.908

Vht: $\quad-263.83 \quad-109.21 \quad-79.78$

Fig. 15. Walén relation (left) and deHoffmann-Teller frame (right) between 07:54-08:24 UT measured by sc3.

\section{Cluster observations between 07:50-09:00 UT during ortho-Parker spiral, small negative $b_{z}$}

After 07:45 UT the IMF rotates southward followed by a rotation of $b_{x}$ such that in $x, y$-plane the IMF has an orthoparker spiral orientation. The bottom panel of Fig. 2 shows that between 08:00-08:15 UT there are patches of magnetosheath energy ions in regions of tenuous plasma similar to what was observed between 05:30-06:00 UT. There is also mixed (magnetospheric and magnetosheath energies) plasma present between $\approx 08: 06-08: 09$ UT. At 08:00 UT the IMF is dominantly pointing southward, so that reconnection can operate in the sub-solar region but also between the magnetosheath and magnetospheric fields at the flank. Figure 14 (same format as Fig. 5) shows plasma (left) and magnetic field (right) observations between 07:54-08:24 UT in the boundary normal coordinates of sc3. At $\sim 08: 10$ UT all 4 spacecraft are observing a strong bipolar signature in the normal component of the magnetic field together with a local total pressure enhancement, consistent with a flux transfer event like structure (Paschmann et al., 1982). Compared to the continuous train of bipolar variations of the normal component observed between 05:00-06:00 UT, this seems to be a more isolated structure. The Walén relation (left) and deHoffmann-Teller frame (right) between 07:54-08:24 UT measured by sc3 for this interval is shown in Fig. 15. The Walén relation is poor and, compared to the interval between 05:30-06:00 UT that had an excellent HT-frame (slope $\sim 0.93$ and cc. $\sim 0.96$.), the HT-frame slope is only 0.83 and correlation coefficient 0.91 . Figure 16 is a zoom onto the FTE, indicating that the spacecraft are crossing different regions of the FTE. The boundary normal obtained with MFRmethod (for sc3) with $\lambda_{1} / \lambda_{2}=2.2$ is pointing almost entirely toward positive $z$-direction with a small negative $x$ - and $y$ component (upper right corner). $\mathrm{Sc} 1$ is also observing a strongly northward normal. Figure 17 is showing the Walén test and HT-frame measured by sc 3 for this interval. Even for this shorter interval the Walén relation is poor, which indicates that this is not a rotational discontinuity. Perhaps this bipolar signature could be caused by the pressure enhancement after 08:00 UT (Fig. 1). Variations of the solar wind dynamic pressure can generate a wavelike motion along the magnetospheric boundary and cause bipolar signatures of the normal component of the magnetic field (Sibeck et al., 1989; Sibeck, 1992; Otto, 1995). One possibility also might be the deviation from one-dimensionality or stationarity that are required for the relation to strictly hold. Other possibility is that this is a flux rope reconnected some time in the past, but it is not clear why the Walén test would fail to succeed in 

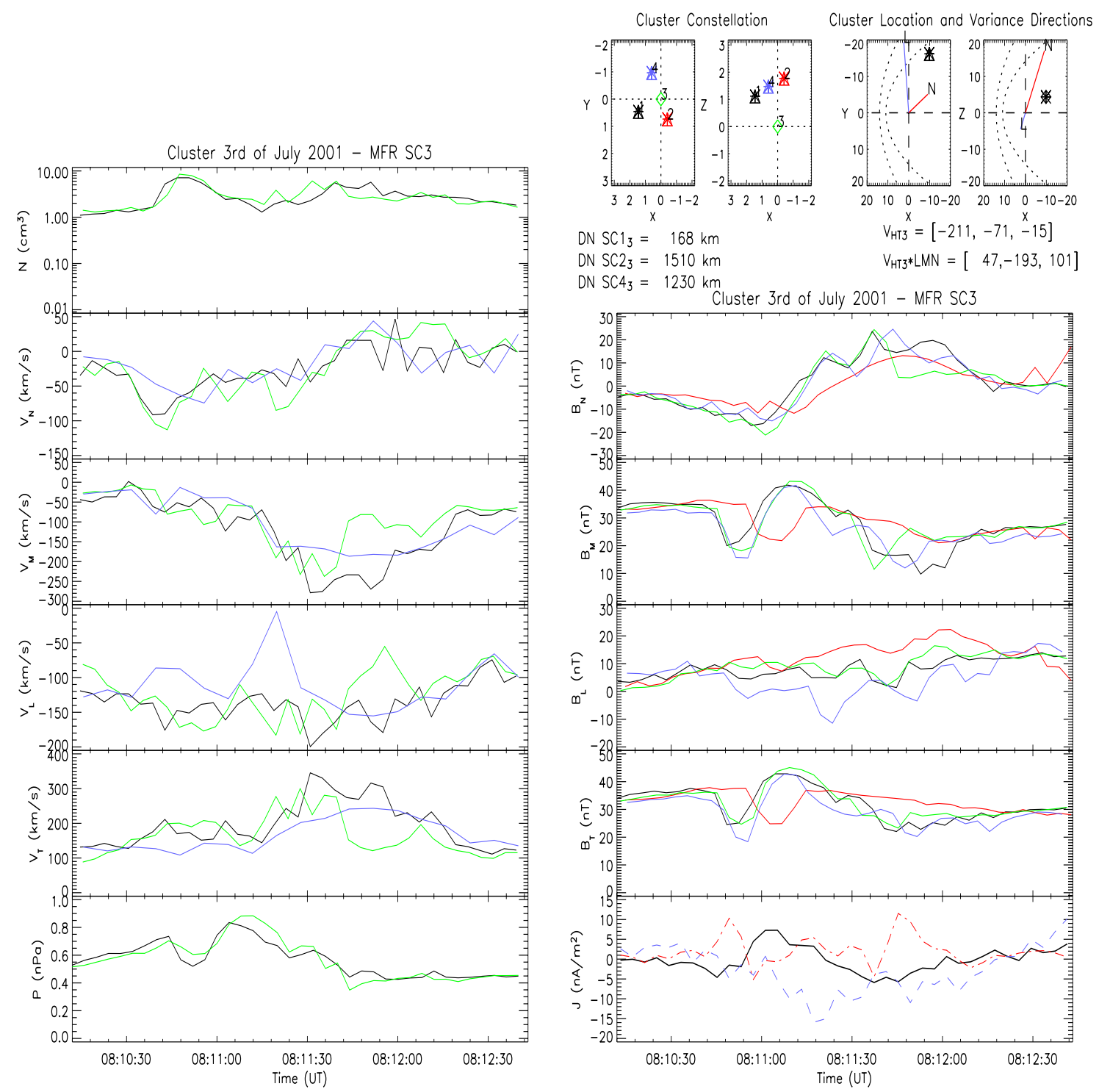

Fig. 16. Plasma (left) and magnetic field (right) observations between 08:10:12-08:12:45 UT in the boundary normal coordinates of sc3. Figure uses same format as Fig. 5.

this case. Sonnerup et al. (2004) suggest that such a FTE could be a "fossil" structure originating from reconnection site that has become inactive. For this shorter interval sc3 observes better but not very good HT-frame with slope of 0.89 and cc. of 0.95 . Also sc1 has a poor Walén relation for this interval, but contrarily to sc 3 it observes a good HTframe with slope of 0.95 and cc. of 0.98 moving with a velocity of $v_{\mathrm{HT}}=[-241,-93,-32] \mathrm{km} / \mathrm{s}$. Although the intervals 05:30-06:00 UT and 08:00-08:15 UT have similar plasma characteristics showing magnetosheath population inside the magnetopause, the source region for plasma entry seems to be different. Between 05:30-06:00 UT Cluster encountered thin current layers with ongoing reconnection due to KHI, whereas during 08:00-08:15 UT the spacecraft encountered a flux rope like signature either due to the pressure pulse or from a possibly remote reconnection site. Furthermore, the HT-frame velocities were very different for the two cases. We propose that (if not due to the pressure pulse) this isolated flux rope is a result of reconnection between northward pointing magnetospheric field and southward pointing IMF field along the dawn flank boundary, or maybe in the more dayside regions.

\section{Discussion and conclusions}

The presented results indicate that during 3 July 2001, Cluster spacecraft had several crossings between hot and tenuous magnetospheric plasma and cold and dense magnetosheath 

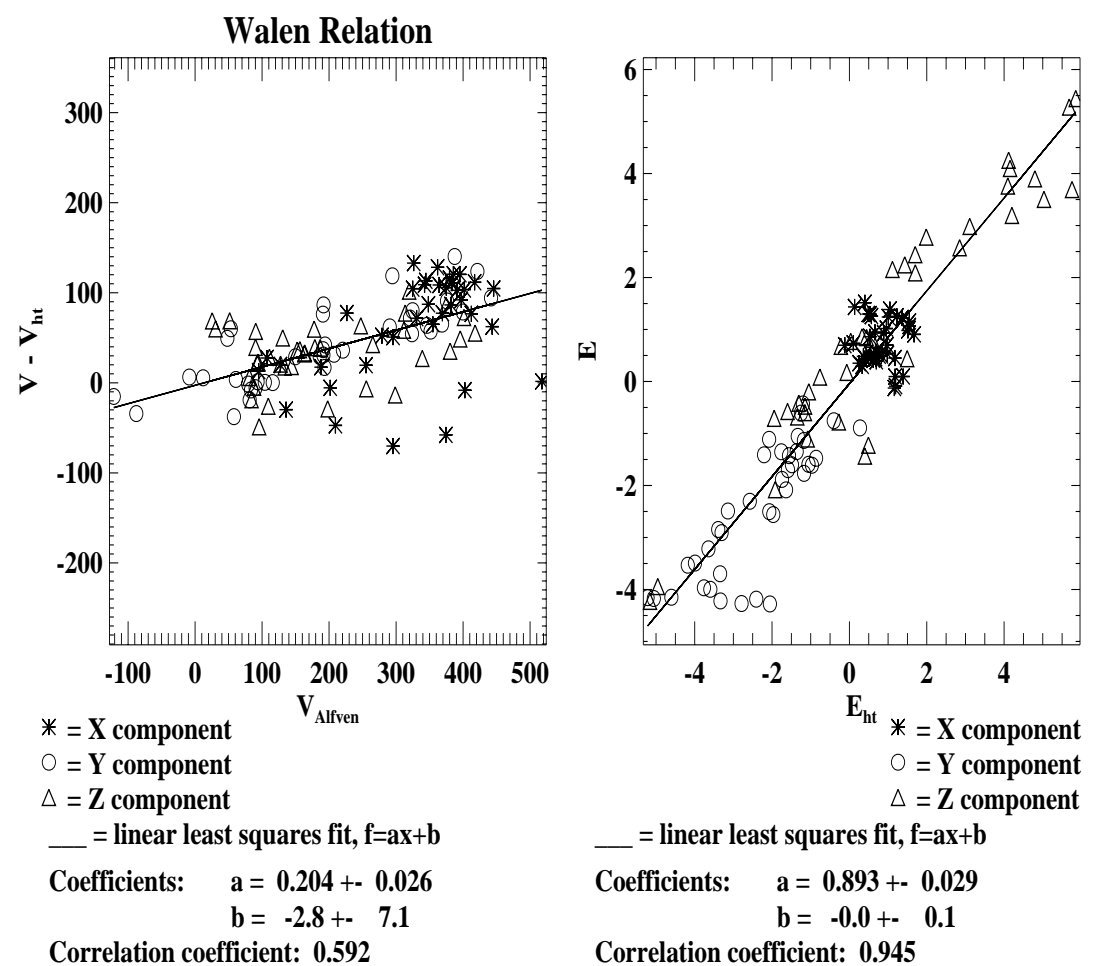

$\ldots$ _ linear least squares fit, $\mathrm{f}=\mathrm{ax}+\mathrm{b}$

Coefficients: $\quad a=0.893+0.029$

$\mathrm{b}=-0.0+-0.1$

Correlation coefficient: $\mathbf{0 . 9 4 5}$

Vht: $\quad-211.16 \quad-71.63 \quad-15.28$

Fig. 17. Walén relation (left) and deHoffmann-Teller frame (right) between 08:10:12-08:12:45 UT determined from sc3 measurements.

plasma. As the spacecraft traversed at the dawnside magnetospheric flank they observed for $1 \mathrm{~h}$ systematic bipolar variations of the normal component of the magnetic with a period of 1-4 min. The leading edge of the bipolar variation showed a more rapid transition indicative of very thin magnetic boundaries, whereas the trailing edge transition was slower. This signature is presumably caused by KelvinHelmholtz instability (KHI) operating at the dawnside magnetospheric flank.

At the first instant the occurrence of the KHI seems surprising because of the prevailing solar wind conditions. Namely the IMF formed a Parker-spiral with a small $b_{z}$ component, which generates a strong tangential magnetic field along the shear flow layer when draped around the magnetopause, and thus stabilises the KH in this plane. We tested the observed configuration with 2-D MHD simulations, and indeed the KH was stable in the shear flow plane.

In Cluster data we found evidence of quasi-periodic boundary normal oscillations showing most variations in $z-$ direction. The deHoffmann-Teller frame velocities had a significant $z$-component, and also the actual plasma velocity had a $z$-component. These observations support the existence of a $\mathrm{KH}$ mode with $k$-vector out from the equatorial plane. We estimated the $\mathrm{KH}$ wavelength to be $6 R_{E}$ from the deHoffmann-Teller frame velocity and from the $\sim 2.2 \mathrm{~min}$ period of the bipolar variation of the normal component of the magnetic field and taking into account that $k$-vector is out from the equatorial plane. Based on these observations we constructed 2-D MHD simulations with the $k$-vector out from the shear flow plane, and in this geometry, the KHI can grow and can generate reconnection in two locations (see Fig. 11) within the Kelvin-Helmholtz wave:

A) at the boundary between anti-parallel magnetosheath and magnetospheric field, where the twisting of the boundary by KHI enables reconnection. Without slight twisting of the boundary this would not be possible because shear flow stabilizes the tearing mode.

B) in the low density part of the wave in a layer of positive current, which is generated when $\mathrm{KH}$ twists the magnetospheric field.

In the Cluster data sc3 and sc4 observations show evidence of the type B)-reconnection. Also, location of the sc1 reconnection interval matches the crossing of the flux tube originating from type A) reconnection site. Our simulations were able to reproduce many of the observed signatures in the satellite data: the bipolar variation of the normal component of the magnetic field and velocity; the density, temperature and pressure variation; the properties and location of 
the intervals satisfying good Walén relation and HT-frame. Although both the KH wave and FTE show bipolar variations of the normal component of the magnetic field, one can distinguish between them by looking at the total pressure: Paschmann et al. (1982) showed that in case of a FTE the total pressure is strongly enhanced, whereas in case of the $\mathrm{KH}$-wave, the total pressure is decreased during the enhanced normal component of the magnetic field (see Fig. 11).

Also the timing of the intervals matches the spacecraft separation and location within the wave: For example, sc4 locates furthest toward the magnetosheath, where the tangential distance across the magnetospheric current layer is shorter (it can only be picked by testing a short $40 \mathrm{~s}$ interval for sc4 (5 data points for sc4)). Sc3, which locates more on the magnetospheric side of the vortex observes a good Walén relation and HT-frame for a one minute! In simulation box this corresponds to a distance of $1.1 R_{E}$.

These observed reconnection intervals must be only a subset of all reconnection events because if they cross a spacecraft at a steeper angle the crossing would only take a few seconds and they would not be identified. Also, the current layers earthward of the type A) reconnection site are much thinner and almost impossible to observe with the current data resolution.

After the IMF turned southward Cluster also observed magnetosheath plasma inside the magnetosphere and an isolated flux rope like signature, but the analysis showed that this might have been a 'fossil' flux tube, which did not satisfy the Walén relation.

The present results indicate that KHI with a $k$-vector with a $z$-component can trigger reconnection at the dawnside flank magnetopause during a Parker-spiral orientated IMF with a small $b_{z}$-component. In Cluster data we found magnetosheath energy ions in the tenuous magnetospheric-like plasma during the intervals when reconnection criteria was met, so that some mixing due to this mechanism may occur. However, it is presently unclear whether reconnection in this configuration leads to significant plasma transport into the magnetosphere in contrast to the cases where reconnection occurs in the high density region due to the twisting of the initially parallel fields by the KHI (Nykyri and Otto, 2001). Based on the simulation result of Fig. 11, it seems that magnetosheath plasma may get into the magnetospheric field lines only due to the type A)-reconnection. However, transport may also occur in the opposite direction due to the type B) reconnection (magnetospheric plasma is captured within higher density part of the vortex).

In our future work we will compare in detail the observations of this day with 3-D MHD simulations in appropriate geometry. In 3-D simulations we will study the significance of plasma transport due to this mechanism and its effectiveness in formation of the cold dense plasma sheet. Particularly important would be to study the statistical occurrence of the KHI in the dawn and duskside flanks for Parker-spiral (PS) and ortho-Parker spiral (OPS) IMF orientations. For
PS (OPS) orientation the dawnside (duskside) flank is more unstable than the dusk (dawn) flank for the KHI in the shear flow plane which may result in asymmetries in the cold dense plasma sheet formation. However, as demonstrated in this paper it is possible (for the stable configuration in shear flow plane) for the modes with $k$-vectors out from the shear-flow plane to grow. Therefore, it is first essential to understand the 3-D dynamics (and resulting magnetic field topology) of reconnection generated by $\mathrm{KHI}$ for these configurations in dawn and dusk-side flanks.

Acknowledgements. We acknowledge ACE SWEPAM and MAG instrument teams for the use of level 2 data, and PPARC for funding for K. Nykyri. Work at Los Alamos was conducted under the auspices of the U.S. Department of Energy, with support from NASA Cluster program. We thank B. Sonnerup for a comment on the HTframes. We would also like to thank S. Schwartz for helpng us to compare our MFR results with the results of Queen Mary MFR software. We would also like to thank all Cluster FGM and CIS team members for making this study possible.

Topical Editor I. A. Daglis thanks G. Paschmann and another referee for their help in evaluating this paper.

\section{References}

Balogh, A., Dunlop, M. W., Cowley, S. W. H., Southwood, D. J., Thomlinson, J. G., et al.: The Cluster Magnetic Field Investigation, Space Sci. Rev., 79, 65-91, 1997.

Balogh, A., Carr, C. M., Acuna, M. H., Dunlop, M. W., Beek, T. J., Brown, P., Fornangon, K.-H., Georgescu, E., Glassmeier, K.-H., Harris, J., Musmann, G., Oddy, T., and Schwingenschuh, K.: The Cluster magnetic field investigation: overview of in-flight perfomance and initial results, Ann. Geophys., 19, 1207-1217, 2001, http://www.ann-geophys.net/19/1207/2001/.

Birn, J.: Computer studies of the dynamical evolution of the geomagnetic tail, J. Geophys. Res., 85, 1214-1222, 1980.

Borovsky, J. E., Thomsen, M. F., and Elphic, R. C.: The driving of the plasma sheet by the solar wind, J. Geophys. Res., 103 , 17 617-17 640, 1998.

Brackbill, J. U. and Knoll, D. A.: Transient magnetic reconnection and unstable shear layers, Phys. Rev. Lett., 86, 2329-2332, 2001.

Chen, Q., Otto, A., and Lee, L. C.: Tearing instability, KelvinHelmholtz instability, and magnetic reconnection, J. Geophys. Res., 102, 151-161, 1997.

Fairfield, D. H., Lepping, R. P., Hones, E., Bame, S., and Asbridge, J.: Simultaneous measurements of magnetotail dynamics by IMP spacecraft, J. Geophys. Res., 86, 1396-1414, 1981.

Fairfield, D. H., Otto, A., Mukai, T., Kokubun, S., Lepping, R. P., Steinberg, J. T., Lazarus, A. J., and Yamamoto, T.: Geotail observations of the Kelvin-Helmholtz instability at the eqatorial magnetotail boundary for parallel northward fields, J. Geophys. Res., 105, 21 159-21 174, 2000.

Fujimoto, M. and Terasawa, T.: Anomalous ion mixing within an MHD scale Kelvin-Helmholtz vortex, J. Geophys. Res., 99, 8601-8614, 1994.

Fujimoto, M. and Terasawa, T.: Anomalous ion mixing within an MHD scale Kelvin-Helmholtz vortex, 2, Effects of inhomogeneity, J. Geophys. Res., 100, 12 025-12 034, 1995. 
Fujimoto, M., Terasawa, T., Mukai, T., Saito, Y., Yamamoto, T., and Kokubun, S.: Plasma Entry from the flanks of the near-Earth magnetotail: Geotail observations, J. Geophys. Res., 103, 43914408, 1998.

Fujimoto, M., Mukai, T., Matsuoka, A., Saito, Y., Hayakawa, H., Kokubun, S., and Lepping, R. P.: Multi-point observations of cold-dense plasma sheet and its relation with tail-LLBL, Adv. Space Res., 25, 1607-1616, 2000.

Haaland, S. E., Sonnerup, B. U. ., Dunlop, M. W., Balogh, A., Georgescu, E., Hasegawa, H., Klecker, B., Paschmann, G., PuhlQuinn, P., Rème, H., Vaith, H., and Vaivads, A.: Four-spacecraft determination of magnetopause orientation, motion and thickness: comparison with results from single-spacecraft methods, Ann. Geophys., 22, 1347-1365, 2004, http://www.ann-geophys.net/22/1347/2004/.

Hasegawa, H., Fujimoto, M., Phan, T.-D., Rème, H., Balogh, A., Dunlop, M. W., Hashimoto, C., and TanDokoro, R.: Transport of solar wind into Earth's magnetosphere through rolled-up KelvinHelmholtz vortices, Nature, 430, 755-758, 2004.

Khrabrov, A. V. and Sonnerup, B.: Orientation and motion of current layers: Minimization of the Faraday residue, Geophys. Res. Lett., 25, 2373-2376, 1998.

Lavraud, B., Thomsen, M. F., Taylor, M. G. G. T., Wang, Y. L., Phan, T. D., Schwartz, S. J., Elphic, R. C., Fazakerley, A., Rème, H., and Balogh, A.: Characteristics of the magnetosheath electron boundary layer under northward interplanetary magnetic field: Implications for high-latitude reconnection, J. Geophys. Res., 110, A06209, doi:10.1029/2004JA010 808, 2005.

Lee, L. C. and Olson, J. V.: Kelvin-Helmholtz instability and the variation of geomagnetic pulsation activity, Geophys. Res. Lett., 7, 777-780, 1980.

Lennartsson, W.: A scenario for solar wind penetration of the Earth's magnetic tail based on ion composition data from the ISEE 1 spacecraft, J. Geophys. Res., 97, 19221-19238, 1992.

Li, W., Raeder, J., Dorelli, J., Oieroset, M., and Phan, T. D.: Plasma sheet formation during long period of northward IMF, Geophys. Res. Lett., 32, L12S08, doi:10.1029/2004GL021524, 2005.

Nikutowski, B., Büchner, J., Otto, A., Kistler, L. M., Mouikis, C., Haerendel, G., and Baumjohann, W.: Equator-S observations of reconnection coupled to surface waves, Adv. Space Res., 29(7), 1129-1134, 2002.

Nykyri, K.: Influence of the Kelvin-Helmholtz instability on the plasma transport at the magnetospheric boundary, Phd thesis, University of Alaska, 2002.

Nykyri, K. and Otto, A.: Plasma Transport at the Magnetospheric Boundary due to Reconnection in Kelvin-Helmholtz Vortices, Geophys. Res. Lett., 28, 3565-3568, 2001.

Nykyri, K. and Otto, A.: Influence of the Hall term on KH instability and reconnection inside KH vortices, Ann. Geophys., 22, 935-949, 2004, http://www.ann-geophys.net/22/935/2004/.

Nykyri, K., Otto, A., J. Büchner, Nikutowski, B., Baumjohann, W., Kistler, L. M., and Mouikis, C.: Equator-S Observations of Boundary Signatures: FTE's or Kelvin-Helmholtz Waves?, Geophysical Monograph, 133, Earth's Low Latitude Boundary Layer, 205-210, 2003.

Ohtani, S., Rostoker, G., Takahashi, K., Angelopoulos, V., Nakamura, M., Waters, C., Singer, H., Kokobun, S., Tsuruda, K., Hughes, W. J., Potemra, T. A., Zanetti, L. J., Gary, J. B., Lui, A.
T. Y., and Williams, D. J.: Coordinated ISTP satellite and ground observations of morningside Pc5 waves, J. Geophys. Res., 104, 2381-2398, 1999.

Oieroset, M., Raeder, J., Phan, T. D., Wing, S., McFadden, J. P., Li, W., Fujimoto, M., Rème, H., and Balogh, A.: Global cooling and densification of the plasma sheet during an extended period of purely northward IMF on October 22-24, 2003, Geophys. Res. Lett., 32, L12S07, doi:10.1029/2004GL021523, 2005.

Olson, J. V.: ULF signatures of polar cusp, J. Geophys. Res., 91, 10 055-10 062, 1986.

Olson, J. V. and Rostoker, G.: Longitudinal phase variations of $P_{c}$ 4-5 micropulsations, J. Geophys. Res., 83, 2481-2488, 1978.

Onsager, T. G., Scudder, J. D., Lockwood, M., and Russell, C. T.: Reconnection at the high-latitude magnetopause during northward interplanetary magnetic field conditions, J. Geophys. Res., 106(A11), 25 467-25 488, 2001.

Otto, A.: 3D resistive MHD computations of magnetospheric physics, Comput. Phys. Commun., 59, 185-195, 1990.

Otto, A.: Properties of pressure pulses and magnetic reconnection at the dayside magnetopause, in: Physics of the Magnetopause, Geophys. Monogr. Ser., vol. 90, edited by: Sonnerup, B. and Song, P., p. 303, AGU, Washington, D.C., 1995.

Otto, A. and Fairfield, D. H.: Kelvin-Helmholtz instability at the magnetotail boundary: MHD simulation and comparison with Geotail observations, J. Geophys. Res., 105, 21 175-21 190, 2000.

Paschmann, G., Haerendel, G., Papamastorakis, I., Sckopke, N., Bame, S. J., Gosling, J., and Russell, C. T.: Plasma and magnetic field characteristics of magnetic flux transfer events, J. Geophys. Res., 82, 2159-2168, 1982.

Paschmann, G., Haaland, S., Sonnerup, B. U. O., Hasegawa, H., Georgescu, E., Klecker, B., Phan, T. D., Réme, H., and Vaivads, A.: Characteristics of the near-tail dawn magnetopause and boundary layer, Ann. Geophys., 23, 1481-1497, 2005, http://www.ann-geophys.net/23/1481/2005/.

Potter, D. E.: Computational Physics, John Wiley, New York, 1973.

Rème, H., Aoustin, C., Bosqued, J. M., Dandouras, I., Lavraud, B., and et. al.: First multispacecraft ion measurements in and near the Earth's magnetosphere with the identical Cluster ion spectrometry (CIS) experiment, Ann. Geophys., 19, 1303-1354, 2001,

http://www.ann-geophys.net/19/1303/2001/.

Russell, C. T., Le, G., and Petrinec, S. M.: Cusp observations of high-and low-latitude reconnection for northward IMF: An alternate view, J. Geophys. Res., 105, 5489-5495, 2000.

Sibeck, D. G.: Transient events in the outer magnetosphere: Boundary waves or FTEs?, J. Geophys. Res., 97, 4009-4026, 1992.

Sibeck, D. G., Baumjohann, W., Elphic, R., Fairfield, D., et al.: The magnetospheric response to 8-minute period, strong-amplitude upstream pressure variations, J. Geophys. Res., 94, 2505-2519, 1989.

Song, P. and Russell, C. T.: Model for the formation of the lowlatitude boundary layer for strongly northward interplanetary magnetic field, J. Geophys. Res., 97, 1411-1420, 1992.

Sonnerup, B. U. Ö., Paschmann, G., Papamastorakis, I., Sckopke, N., Haerendel, G., Bame, S. J., Asbridge, J. R., Gosling, J. T., and Russell, C. T.: Evidence for magnetic reconnection at the earth's magnetopause, J. Geophys. Res., 86, 10 049-10 067, 1981.

Sonnerup, B. U. Ö., Paschmann, G., and Phan, T.-D.: Fluid aspects 
of reconnection at the magnetopause: In situ observations, in: Physics of the Magnetopause, Geophys. Monogr. Ser., vol. 90, edited by: Song, P., Sonnerup, B. U. Ö., and Thomsen, M. F., p. 167, AGU, Washington, D.C., 1995.

Sonnerup, B. U. Ö., Hasegawa, H., and Paschmann, G.: Anatomy of a flux transfer event seen by Cluster, Geophys. Res. Lett., 31, L11803, doi:10.1029/2004GL020134, 2004.
Terasawa, T., Fujimoto, M., Mukai, T., Shinohara, I., Saito, Y., Yamamoto, T., Machida, S., Kokubun, S., Lazarus, A. J., Steinberg, J. T., and Lepping, R. P.: Solar wind control of density and temperature in the near-Earth plasma sheet: WIND/Geotail collaboration, Geophys. Res. Lett., 24, 935-938, 1997.

Thomas, V. A. and Winske, D.: Kinetic simulations of the KelvinHelmholtz instability at the magnetopause, J. Geophys. Res., 98, 11425-11 438, 1993. 